$\underline{\text { Preprint typeset in JHEP style. - HYPER VERSION }}$

ATLAS-COM-PHYS-2002-034

Cavendish HEP-2002-02/11

CERN-TH/2002-190

DAMTP-2002-104

\title{
Discovering anomaly-mediated supersymmetry at the LHC
}

\author{
A.J. Barr, C.G. Lester, M.A. Parker \\ Cavendish Laboratory, University of Cambridge, Madingley Road, Cambridge, \\ CB3 OHE, UK. \\ B.C. Allanach \\ Theory Division, CERN, 1211 Geneva 23, Switzerland.

\section{P. Richardson} \\ DAMTP, Silver Street, Cambridge CB3 9EW, UK, and \\ Cavendish Laboratory, University of Cambridge, Madingley Road, Cambridge, \\ CB3 OHE, UK.
}

\begin{abstract}
The discovery potential of the LHC is investigated for the minimal anomaly-mediated supersymmetry breaking (mAMSB) scenario, using the ATLAS fast detector simulator, including track reconstruction and particle identification. Generic supersymmetry search cuts are used to map the $5 \sigma$ (and $\geq 10$ event) discovery contours in the $m_{0}-m_{3 / 2}$ plane. With $100 \mathrm{fb}^{-1}$ of integrated luminosity the search will reach up to $2.8 \mathrm{TeV}$ in the squark mass and $2.1 \mathrm{TeV}$ in the gluino mass. We generalise a kinematical variable and demonstrate that it is sensitive to the small chargino-LSP mass splitting characteristic of AMSB models. By identifying tracks from chargino decays we show that the Wino-like nature of the LSP can be determined for a wide range of chargino lifetimes.
\end{abstract}

KEYWORDS: Supersymmetry Breaking, Supersymmetric Standard Model, Hadronic Colliders. 


\section{Contents}

1. Introduction 2

2. Benchmark points 3

3. The ATLAS detector 6

4. LHC reach for mAMSB 6

4.1 Event simulation 9

4.2 Optimisation of cuts 10

4.3 Results 13

5. Distinguishing Wino-like LSPs 13

5.1 Definition of points 15

5.2 Identifying chargino decay products 19

5.3 Results 21

6. Other constraints 24

6.1 Cosmological relic density 24

6.2 Muon $g-2 \quad 26$

$6.3 B \rightarrow X_{s} \gamma$

7. Conclusions 27

A. The variable $m_{T 2}$ and its generalisation 30

A.1 The properties of $m_{T 2}$

A.2 Generalisations of $m_{T 2}$

A.3 Uncertainties in $\boldsymbol{p}_{T}$ and $m_{\tilde{\chi}_{1}^{0}}$

B. Simulation of particle tracks 36

B.1 Low $p_{T}$ electron identification 36

B.2 Low $p_{T}$ muon identification 37 


\section{Introduction}

Weak scale supersymmetry (SUSY) is the most promising solution to the technical hierarchy problem of the Standard Model (SM). However supersymmetric partners of known particles have not yet been discovered by experiment so if SUSY exists it must be broken. Dynamical supersymmetry breaking is usually assumed to occur in a 'hidden sector' which is isolated from ordinary particles and interactions. It is then communicated to the fields of the minimal supersymmetric standard model (MSSM) by some messenger(s). This must be achieved while maintaining both the theoretically appealing aspects such as naturalness $[1,2]$ and respecting experimental constraints on masses, flavour-changing neutral currents and $\mathrm{CP}$ violation.

SUSY breaking must be communicated to the observed particles by some interaction felt by both the visible and hidden sectors. The most commonly discussed methods have been flavour-blind gravitational (SUGRA), or gauge (GMSB) interactions.

More recently an alternative mechanism known as anomaly-mediation (AMSB) has been proposed $[3,4]$ in which a conformal anomaly in the auxiliary field of the supergravity multiplet transmits SUSY-breaking to the observable sector. There will be an anomaly-mediated contribution to the gaugino masses in any hidden-sector model, but where no other direct contribution is present AMSB will be the leading effect.

Anomaly mediation provides a potential solution to the SUSY flavour problem, in a highly predictive model. One undesirable feature of pure anomaly-mediation is that the slepton has a negative mass-squared. There are various ways to solve this problem [3,5-11], the simplest of which is the addition of a universal scalar mass at the GUT scale. The unmeasured parameters of the minimal model (mAMSB) are then $m_{3 / 2}$ - the gravitino mass; $m_{0}$ - the universal scalar mass; $\tan \beta$ - the ratio of the vacuum expectation value of the Higgs fields; and the sign of the $\mu$ parameter multiplying $H_{1} H_{2}$ in the superpotential, the magnitude of which is fixed from the condition of correct electroweak symmetry breaking.

The sparticle spectra for mAMSB have been calculated in [12-15]. With pure anomaly-mediation, (i.e. $m_{0}=0$ ) the gaugino masses are proportional to their beta functions:

$$
M_{i}=\frac{\beta_{g_{i}}}{g_{i}} m_{3 / 2}
$$

where $g_{i}$ are the gauge coupling constants with $i=1,2,3$ indicating the gauge group, $\beta_{g_{i}}$ are their corresponding renormalisation group beta-functions, and $m_{3 / 2}$ is the gravitino mass. AMSB therefore predicts that the gaugino masses are in the approximate ratios $M_{1}: M_{2}: M_{3} \approx 3: 1: 7$ so that the Wino (rather than the more conventional Bino) is the lightest supersymmetric particle (LSP), and the gluino is nearly an order of magnitude heavier than the LSP. 
The prediction in AMSB of a Wino-like LSP has interesting phenomenological consequences. The most striking of these is that the lightest chargino is nearly massdegenerate with the lightest neutralino. Near-degenerate particles are not unusual in SUSY phenomenology, but with AMSB one of these particles is the LSP. Since $\mathrm{R}$-parity is assumed to be conserved in our analysis (unlike e.g. $[16,17]$ ) the $\tilde{\chi}_{1}^{+}$may only decay into the $\tilde{\chi}_{1}^{0}$ so the small mass difference

$$
\Delta M_{\tilde{\chi}_{1}} \equiv m\left(\tilde{\chi}_{1}^{+}\right)-m\left(\tilde{\chi}_{1}^{0}\right)
$$

means that the lightest chargino may have a lifetime long enough to be detected at collider experiments.

In the present paper we examine the AMSB discovery potential of the LHC general-purpose detectors from several points of view. We concentrate on the ATLAS detector but similar considerations will also apply to CMS. In the next section we review the properties of the recently proposed benchmark points for minimal anomaly mediation. In sec. 0 we highlight some of the important features of the ATLAS detector. We explore the potential reach of generic SUSY search techniques in detecting minimal anomaly mediated supersymmetry in sec. 6 . While the generic search reach is dependent on the mAMSB spectrum, the same is not true for the classic anomaly-mediated signature - the Wino-like LSP - which will apply beyond the minimal model. The signatures for identifying Wino-like LSPs at the LHC are

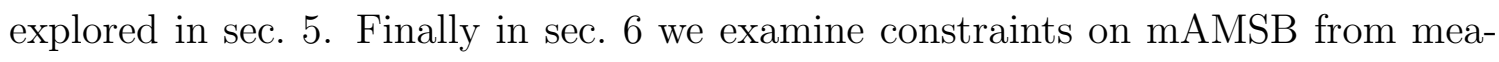
surements of the cosmological relic density, the muon anomalous magnetic moment, and the branching ratio $B \rightarrow X_{s} \gamma$.

\section{Benchmark points}

A set of benchmark points and 'slopes' or model lines was suggested for study at Snowmass [18]. Of the eleven points, only one (SPS 9) applies to mAMSB. That point has the parameters: $m_{0}=450 \mathrm{GeV}, m_{3 / 2}=60 \mathrm{TeV}, \tan \beta=10$, with $\mu>0$, and lies on the model line "slope" $m_{0}=0.0075 \times m_{3 / 2}$, where $m_{3 / 2}$ can vary. Part of the sparticle spectrum for SPS 9 is shown in fig. 1. The lightest sparticles the $\tilde{\chi}_{1}^{+}$and $\tilde{\chi}_{1}^{0}$ - have masses about $170 \mathrm{GeV}$ while squark and gluino masses are about $1.25 \mathrm{TeV}$, so one would expect copious sparticle production at the LHC. The Wino-like character of the lightest chargino and neutralino increases the relative cross-section to non-coloured sparticles as compared to e.g. SUGRA models. Indeed the HERWIG-6.3 [19-21] Monte-Carlo event generator gives the inclusive SUSY crosssection as $3.9 \mathrm{pb}$, of which about $0.5 \mathrm{pb}$ is to squarks and gluinos. The chain $\tilde{q} \rightarrow \tilde{\chi}_{2}^{0} \rightarrow \tilde{l} \rightarrow \tilde{\chi}_{1}^{0}$ is available, ${ }^{1}$ and has a large branching ratio, so good information about the squark, slepton, and at least two neutralino masses could be extracted from kinematic edges in the $l l, l q$ and $l l q$ invariant masses [22-24].

\footnotetext{
${ }^{1}$ Note that SM particles are omitted from decays when this can be done without ambiguity.
} 


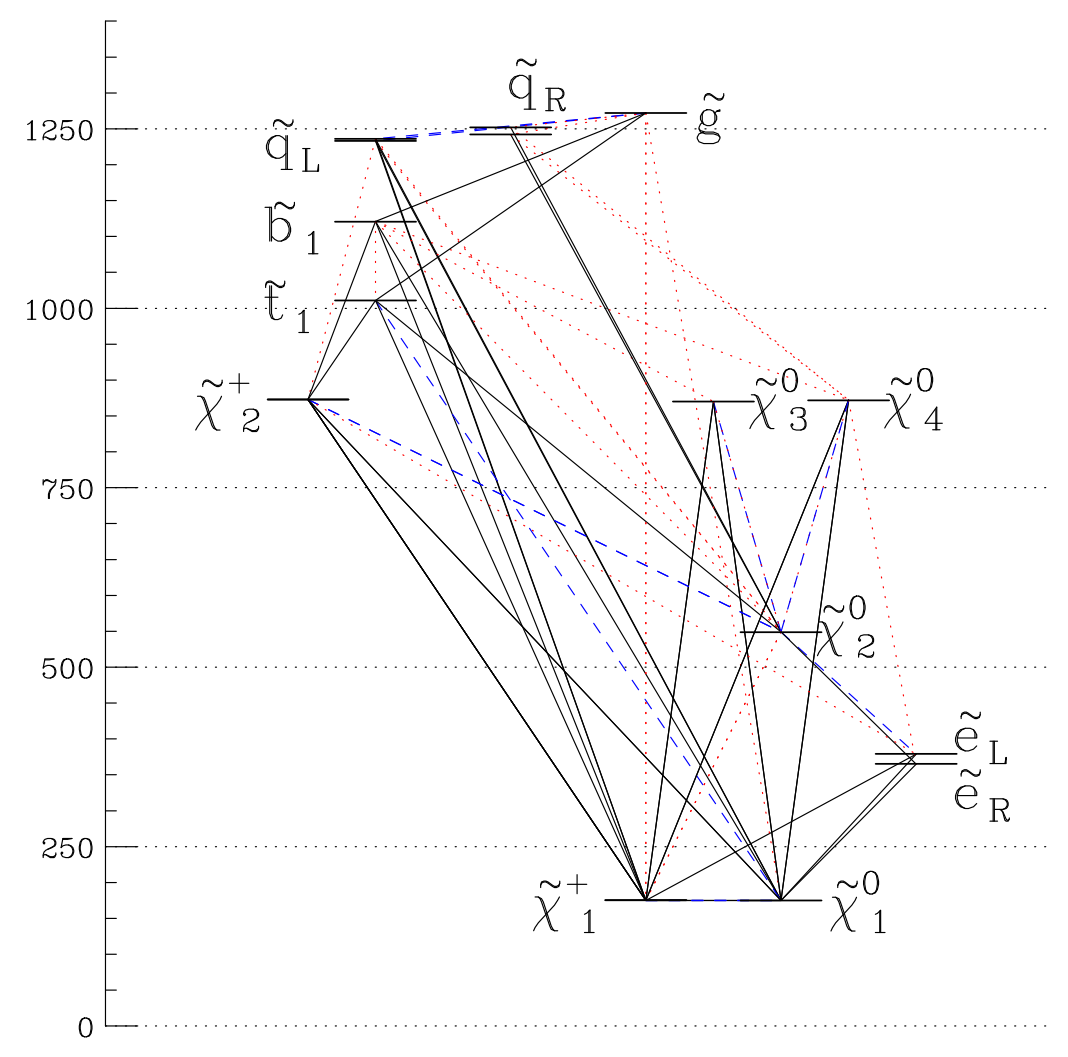

Figure 1: Part of the sparticle spectrum at the Snowmass point SPS 9 which has $m_{0}=$ $450 \mathrm{GeV}, m_{3 / 2}=60 \mathrm{TeV}$ and $\tan \beta=10, \mu>0$. Solid black lines indicate branching ratios (BR's) greater than $10 \%$, dashed blue lines show BR's in the range $1 \% \rightarrow 10 \%$, while red dotted lines show BR's in the range $0.1 \rightarrow 1 \%$. The sparticles are displaced horizontally for clarity.

The $\tilde{e}_{R}$ and $\tilde{e}_{L}$ masses are approximately equal (fig. 1) because in mAMSB the slepton masses are principally determined by $m_{0}$. A more detailed analysis would be required to determine if the $\tilde{e}_{R}$ and $\tilde{e}_{L}$ masses could be separately measured from two nearby edges in the $l l$ invariant mass distribution, as was noted in [24] for a similar point. The decay of the right-sleptons to the Wino-like LSP is suppressed, but not sufficiently to produce a measurable displaced vertex from slepton decay $\left(\tau \sim 10^{-16} \mathrm{~s}\right)$. In fact even if the Bino-like component of the LSP becomes extremely small, the decay $\tilde{e}_{R} \rightarrow \tilde{\tau}_{1} \rightarrow \tilde{\chi}_{1}^{0}$ will remain unsupressed. This is particularly true at high $\tan \beta$ since the mixing between the left- and right-handed staus increases with the tau Yukawa coupling.

Because the gluino has a similar mass to the heavier squarks at SPS 9 it decays primarily to $\tilde{t}_{1}$ and $\tilde{b}_{1}$, meaning that gluino production will lead to large numbers of $b$-quarks. This is not a general (or unique) feature of mAMSB, and while vertex tagging of jets could help distinguish this particular point from the SM it is not used in our analysis. 


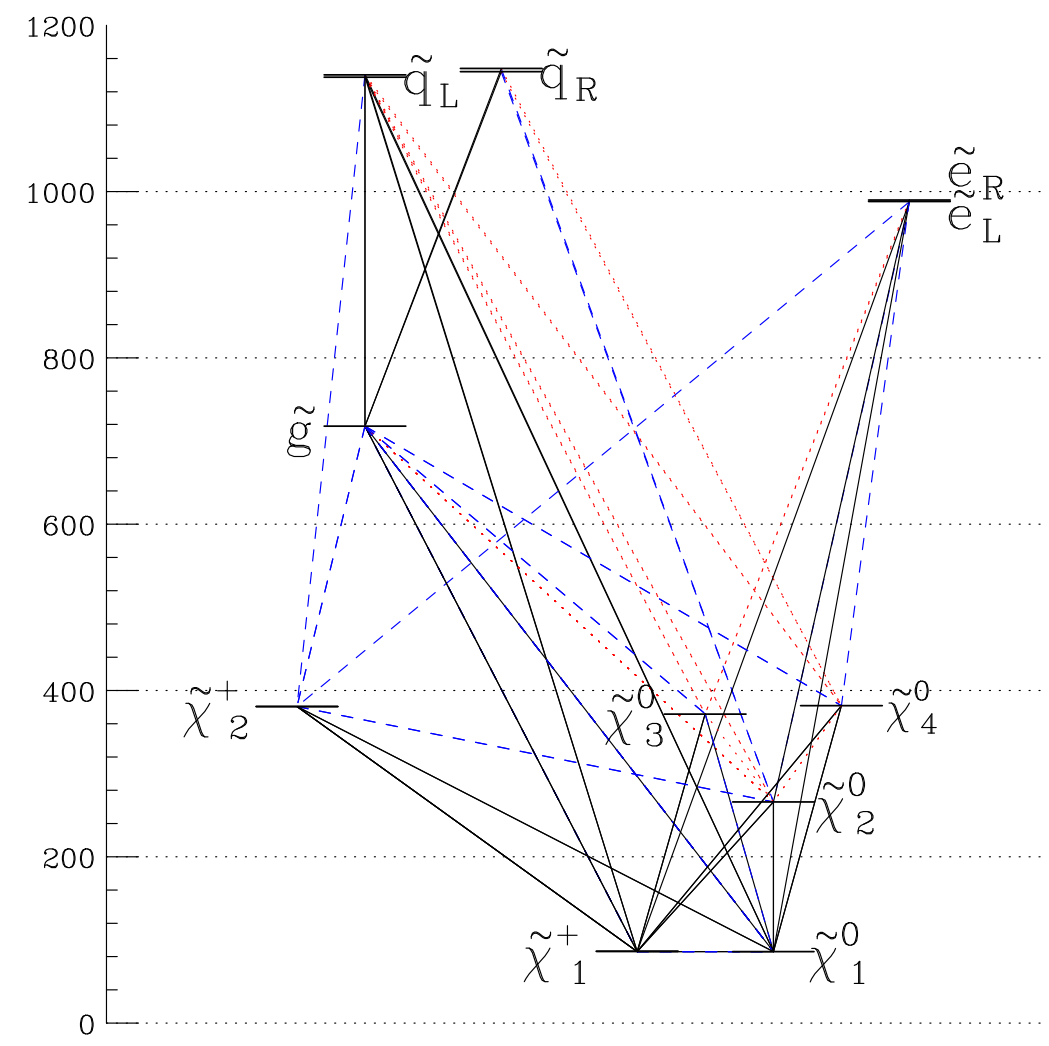

Figure 2: Part of the sparticle spectrum at the Point d'Aix: $m_{0}=1000 \mathrm{GeV}, m_{3 / 2}=$ $30 \mathrm{TeV}, \tan \beta=30, \mu>0$. Branching ratios as for fig. 1. The sparticles are displaced horizontally for clarity.

Another set of benchmarks, the 'Points d'Aix' [25] chooses the mAMSB point $m_{0}=1000 \mathrm{GeV}, m_{3 / 2}=30 \mathrm{TeV}$ also with $\mu>0$, and with the higher value $\tan \beta=30$. With a $\tilde{\chi}_{1}^{+}$mass of $85 \mathrm{GeV}$, this point violates the limits from the LEP search [26] for $e^{+} e^{-} \rightarrow \tilde{\chi}_{1}^{+} \tilde{\chi}_{1}^{-} \gamma$, which places a lower limit on the $\tilde{\chi}_{1}^{+}$mass of about $92 \mathrm{GeV}$ for almost all values of the mass difference $\Delta M_{\tilde{\chi}_{1}}$.

The mAMSB Point d'Aix, despite being ruled out, still has a number of features which are of qualitative interest (fig. 2). The light gluino means that the decays $\tilde{g} \rightarrow \tilde{t} \bar{t}$ and $\tilde{b} \bar{b}$ are no longer kinematically allowed. This means that the major source of $b$ quarks is from $\tilde{\chi}_{2,3,4}^{0} \rightarrow \tilde{\chi}_{1}^{0} h$ followed by $h \rightarrow b \bar{b}$, so fewer heavy quarks are produced than for the Snowmass point. The heavy sleptons can no longer participate in the various chains $\tilde{q} \rightarrow \tilde{\chi}_{x}^{0} \rightarrow \tilde{l} \rightarrow \tilde{\chi}_{y}^{0}$ so slepton mass measurements would be extremely difficult at a hadron collider. There will, however, be large numbers of events in which $\tilde{\chi}_{1}^{ \pm}$s are produced, either directly or from $\tilde{g} \rightarrow \tilde{\chi}_{1}^{ \pm} q \bar{q}$, so a signature based on the near-degeneracy of the $\tilde{\chi}_{1}^{+}$and $\tilde{\chi}_{1}^{0}$ is robust. 


\section{The ATLAS detector}

ATLAS is one of two general-purpose experiments which will explore a wide variety of high $p_{T}$ physics with proton on proton collisions at $\sqrt{s}=14 \mathrm{TeV}$ the LHC. The LHC design luminosity is $\left(10^{34} \mathrm{~cm}^{-2} \mathrm{~s}^{-1}\right)$, with a bunch-crossing period of $25 \mathrm{~ns}$.

The detector design, performance and physics potential are described in [22]. The main components are:

- An inner tracking detector contained within a 2 Tesla solenoidal field which comprises three different elements, each of which has acceptance up to $|\eta|<2.5$ and $2 \pi$ in $\phi$, where $\eta=-\log [\tan (\theta / 2)]$ is the pseudorapidity, $\theta$ is the polar angle from one of the beams, and $\phi$ is the azimuth. In order of increasing radius in the transverse direction $(r$ in $\mathrm{mm}$ ) the sub-detector components are: a pixel detector $(43<r<132)$, a silicon microstrip detector $(300<r<520)$ and a straw-tube transition radiation tracker with electron-identification ability $(560<r<1070)$.

- A highly segmented lead/liquid-argon electromagnetic calorimeter allows electron and photon identification. The hadronic calorimeter employs steel tiles with a scintillator in the barrel region $(|\eta|<1.7)$ with granularity $\Delta \eta \times \Delta \phi=$ $0.1 \times 0.1$, and copper/liquid-argon in the endcaps $(1.5<|\eta|<3.2)$. A dense forward calorimeter with granularity $0.2 \times 0.2$ extends coverage to $|\eta|<5$.

- A muon system consisting of both precision and fast (trigger) detection elements. It is constructed within a toroidal magnet system and extends to transverse radii of about $10 \mathrm{~m}$.

\section{4. $\mathrm{LHC}$ reach for $\mathrm{mAMSB}$}

While some of the phenomenological features are particular to AMSB, one might expect to be able to distinguish AMSB from the SM using the same types of cuts based on leptons, jets and missing energy - that are applied in mSUGRA analyses.

The sensitivity of the LHC to mAMSB has been demonstrated [24] for one point $\left(m_{0}=200 \mathrm{GeV}, m_{3 / 2}=35 \mathrm{TeV}, \tan \beta=3, \mu>0\right)$ with relatively light sparticles, where the sparticle spectrum was investigated in detail. In $[27,28]$, the signatures for AMSB at a future linear $e^{+} e^{-}$collider were investigated. In [29] the reach of the LHC was investigated using a simple generic detector simulation for $10 \mathrm{fb}^{-1}$ of integrated luminosity.

In this section our the aim is to determine the reach of the LHC with a realis-

tic detector simulator, using optimised but generic SUSY cuts and for $100 \mathrm{fb}^{-1}$ of integrated luminosity. 


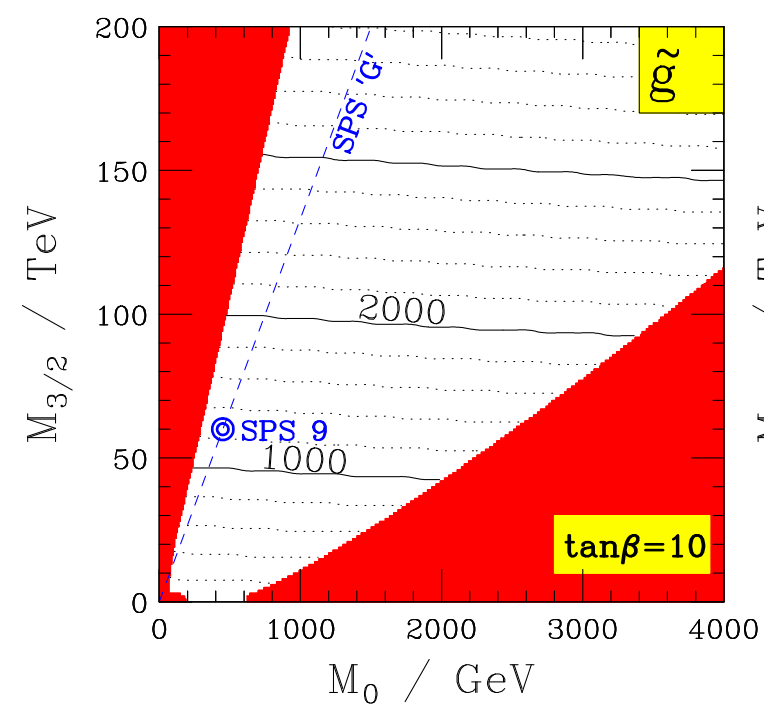

(a)

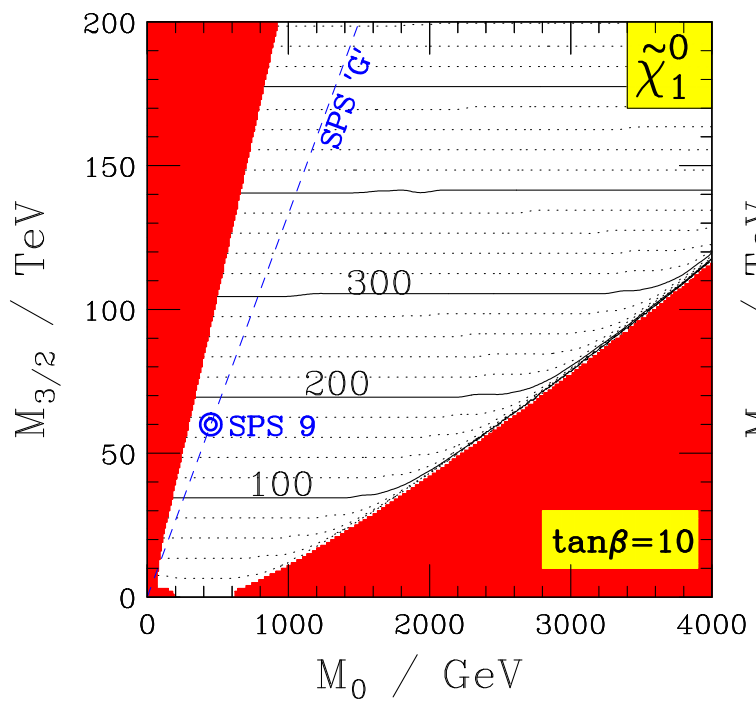

(c)

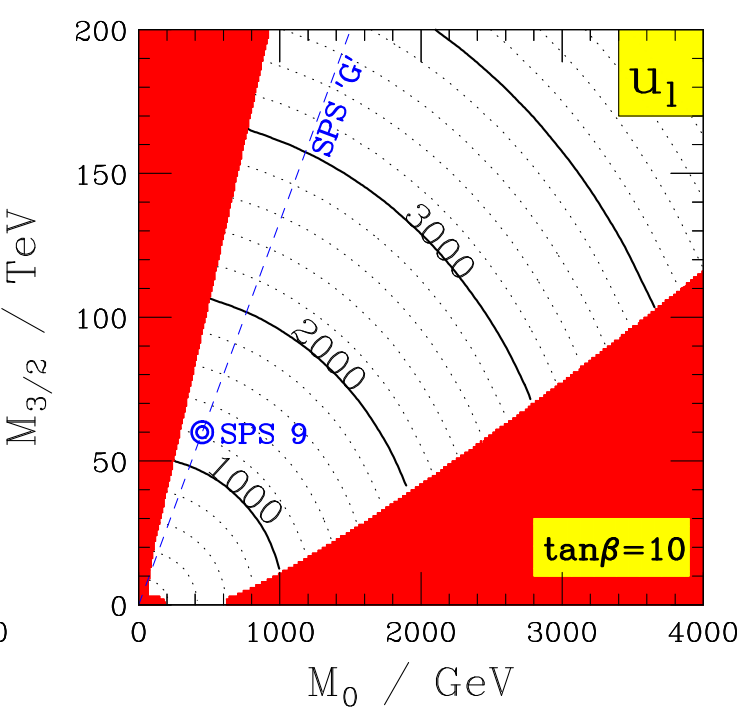

(b)

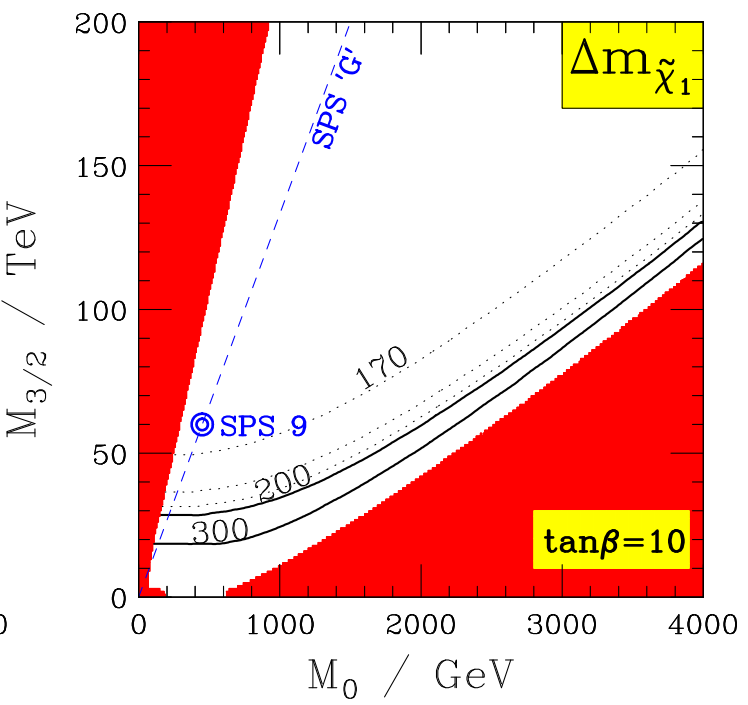

(d)

Figure 3: The contours show the (a) gluino, (b) up left squark, and (c) lightest neutralino mass in $\mathrm{GeV}$, and (d) the $\Delta m=\tilde{\chi}_{1}^{+}-\tilde{\chi}_{1}^{0}$ mass difference in $\mathrm{MeV}$ as a function of $m_{0}$ and $m_{3 / 2}$. The other parameters are: $\tan \beta=10$ and $\mu>0$. The Snowmass point $\left(m_{0}=450 \mathrm{GeV}, m_{3 / 2}=60 \mathrm{TeV}\right)$ is marked as a pair of concentric circles, through which passes the Snowmass slope "model line G" (dashed line). The solid red regions are excluded because of lack of electroweak symmetry breaking (bottom right) or because of charge- or colour-breaking minima, or non- $\tilde{\chi}_{1}^{0}$ LSP (left). $\Delta m$ is greatest where $\mu$ is small, which occurs near to the region where electroweak symmetry is unbroken. 


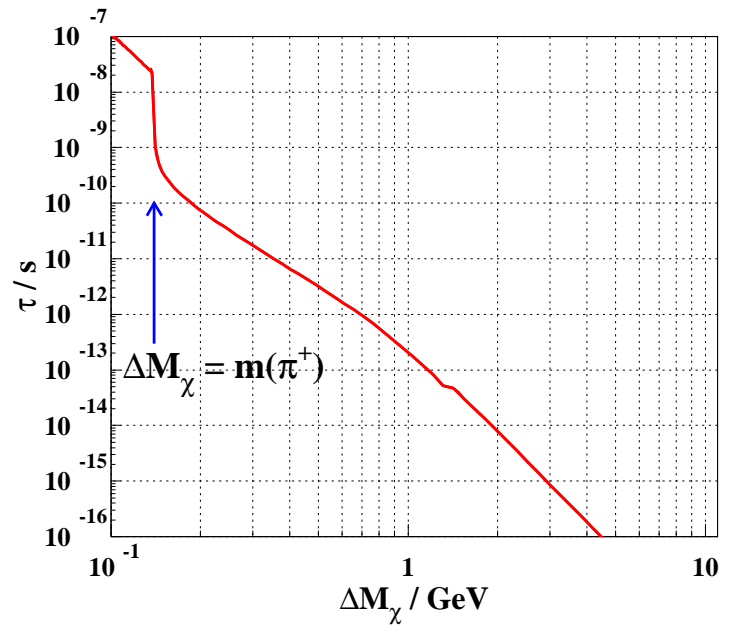

(a)

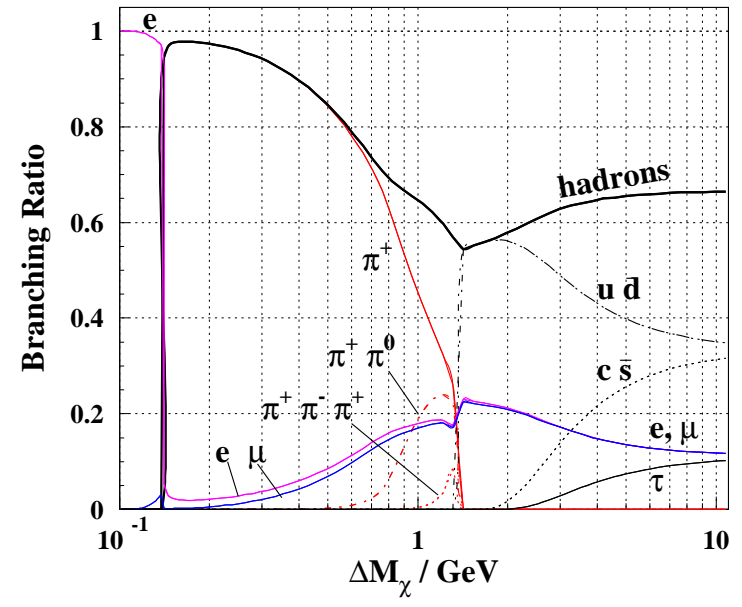

(b)

Figure 4: The (a) lifetime and (b) branching ratios of the lightest chargino as a function of the mass difference $\Delta M_{\chi}=M\left(\tilde{\chi}_{1}^{+}\right)-M\left(\tilde{\chi}_{1}^{0}\right)$. The rapid decrease in the lifetime occurs at $\Delta M_{\chi}=m_{\pi^{+}}$where the single pion mode becomes available. The discontinuity at $\Delta M_{\chi}=1.4 \mathrm{GeV}$ comes from the switch in the calculation from hadronic to partonic decay widths. The leptonic channels implicitly include the corresponding neutrino. The branching ratio to $\pi^{+} \pi^{0} \pi^{0}$ was assumed to be equal to $\pi^{+} \pi^{-} \pi^{+}$. After [30-32]. 


\subsection{Event simulation}

The mAMSB spectra were generated using ISA JET-7.63 [33,34] on a grid $100 \mathrm{GeV} \times$ $5 \mathrm{TeV}$ in the $\left(m_{0}, m_{3 / 2}\right)$ plane. In all cases the ratio of the Higgs vacuum expectation values $(\tan \beta)$ was set equal to 10 and the sign of $\mu$ was positive. The mass of the top quark (important for electroweak symmetry breaking) was taken to be $175 \mathrm{GeV}$ throughout. The dependence of some of the key sparticle masses on the input values of $m_{0}$ and $m_{3 / 2}$ is shown in fig. 3 .

In the $\tilde{\chi}_{1}^{ \pm} \rightarrow \tilde{\chi}_{1}^{0}$ decays, ISAJET does not include masses for the leptons, and does not contain multi-pion decay modes. Since the mass difference $\Delta M_{\tilde{\chi}_{1}}$ can be of the order of the mass of the muon, the lepton mass effects can be important in AMSB. To improve accuracy, the chargino decay modes calculated in $[30,31]$ were implemented with pion form factors from [32] and massive leptons. The resulting chargino lifetime and branching ratios are shown in fig. 4 .

HERWIG-6.3 was used to produce $200 \mathrm{fb}^{-1}$ of unweighted inclusive supersymmetry events for each point (with a minimum of $5 \times 10^{4}$, and up to a maximum of $5 \times 10^{5}$ events). ${ }^{2}$ This was then scaled to give the expected number of events for $\int \mathcal{L}=$ $100 \mathrm{fb}^{-1}$, which corresponds to the first year of 'high luminosity' $\left(10^{34} \mathrm{~cm}^{-2} \mathrm{~s}^{-1}\right)$ running of the LHC. Background samples were generated with HERWIG for the production of $W^{ \pm}+$jets, $Z^{0}+$ jets, $t \bar{t}$, and QCD $2 \rightarrow 2$ (excluding $t \bar{t}$ ). For the $W^{ \pm}+$jets sample, the cross-sections were multiplied by the factor

$$
1.6 \times\left[\frac{m_{W}^{2}+\left(p_{t}^{\mathrm{thr}}\right)^{2}}{m_{W}^{2}}\right]^{2\left(N_{\mathrm{jet}}-1\right)}
$$

where $N_{\text {jet }}$ is the number of jets, and $p_{t}^{\text {thr }}$ is the jet transverse energy threshold which was set to $10 \mathrm{GeV}$. This correction brings the HERWIG cross-section into better agreement with tree-level matrix element calculations [37]. A total of over 20 million background events were generated in logarithmic intervals in the HERWIG parameters PTMIN and PTMAX from 0 to $7000 \mathrm{GeV}$. The background cross-sections are shown before and after preselection cuts in fig. 5 .

The events were passed through the ATLAS fast detector simulator, ATLFAST-2.50 [38], which gives a parameterised detector response based on GEANT3 Monte-Carlo simulations [39]. Jets were found using the ATLFAST cone algorithm with a cone size $\Delta R=0.4$, and a minimum $p_{T}$ of $10 \mathrm{GeV}$. The loss in resolution from pile-up was simulated. Calorimeter cells with $E_{T}$ deposits below $1 \mathrm{GeV}$ were not included in the $\mathbb{E}_{T}$ calculation in order to more accurately model the expected resolution. The cell sizes were $\Delta \eta \times \Delta \phi=0.1 \times 0.1$ for $|\eta|<3$ and $0.2 \times 0.2$ for $3<|\eta|<5$. Otherwise the default ATLFAST parameters were applied. The parameterised tracking simulation used is described in appendix $B$.

\footnotetext{
${ }^{2}$ No significant differences were found on comparing with HERWIG-6.4 [35] which includes spin correlations as described in [36].
} 


\subsection{Optimisation of cuts}

Cuts were applied to leptons (electrons and muons only), jets, and missing transverse energy in a similar manner to those used in [22,29,40-42]. The variables to which cuts were applied were:

(1) $\mathbb{E}_{T}$, missing transverse energy;

(2) $p_{T(J 1)}, p_{T}$ of the hardest jet;

(3) $p_{T(J 2)}, p_{T}$ of the next-to-hardest jet;

(4) $\sum p_{T}$, scalar sum of the $p_{T}$ of jets in the event;

(5) $N_{\text {jet }}$, number of jets in the event;

(6) $S_{\mathrm{T}}$, transverse sphericity (circularity) of the event;

(7) $\Delta \phi_{(J 1)}$, difference in azimuth between hardest jet and $\not_{T}$ vector;

(8) $p_{T(\ell 1)}, p_{T}$ of hardest lepton (if any);

(9) $\Delta \phi_{(\ell 1)}$, difference in azimuth between hardest lepton (if any) and $\boldsymbol{p}_{T}$ vector;

(10) $M_{T}=\sqrt{2 p_{T(\ell 1)} \mathbb{E}_{T}\left(1-\cos \left(\Delta \phi_{(\ell 1)}\right)\right)}$, transverse mass of lepton and missing energy. Applied to single-lepton channel only, to reduce SM leptonic $W^{ \pm}$background.

The preselection cuts which were applied to cut down the background, particularly at low $p_{T}$, are shown in the third column of table 1. The reduction in the background cross-section can be seen in fig. 5. Hard preselection cuts were not placed on isolated electrons or muons since it is foreseen that one jet with $p_{T}>100 \mathrm{GeV}$ together with $\not_{T}>100 \mathrm{GeV}$ will be sufficient to provide the on-line trigger. Other trigger strategies which would improve the reach include:

- Allowing a hard $(\approx 20 \mathrm{GeV})$ isolated lepton as a trigger could improve the selection for points for relatively low $m_{0}$ in which the cascade decay $\tilde{q} \rightarrow \tilde{\chi}_{x}^{0} \rightarrow$ $\tilde{l} \rightarrow \tilde{\chi}_{y}^{0}$ is available;

- Track vertexing which in ATLAS can be applied at the second trigger level could improve the selection for events containing heavy quarks;

- If all of the coloured sparticles are heavy then the dominant production of SUSY particles will be to the lightest gauginos. At leading order in perturbation theory, the production of $\tilde{\chi}_{1}^{0} \mathrm{~s}$ and/or $\tilde{\chi}_{1}^{ \pm} \mathrm{s}$ would be difficult to trigger on, but gaugino production in association with a high $p_{T}$ jet or photon and $\mathbb{E}_{T}$ might be observable $[15,43]$. 


\begin{tabular}{|c|ll|c|c|c|c|c|c|c|c|c|c|}
\hline & \multicolumn{10}{|c|}{ Variable } & \multicolumn{10}{|c|}{ Allowed Values } \\
\hline \hline 1 & $\mathbb{E}_{T}$ & $>$ & $\mathbf{2 0 0}$ & 250 & 300 & 400 & 500 & 600 & 800 & 1000 & 1500 & 2000 \\
2 & $p_{T(J 1)}$ & $>$ & $\mathbf{1 0 0}$ & 150 & 200 & 300 & 400 & 600 & 800 & 1000 & 1500 & 2000 \\
3 & $p_{T(J 2)}$ & $>$ & $\mathbf{1 0 0}$ & 150 & 200 & 300 & 400 & 600 & 800 & 1000 & 1500 & 2000 \\
4 & $\sum p_{T}$ & $>$ & $\mathbf{2 0 0}$ & 250 & 300 & 400 & 500 & 600 & 800 & 1000 & 1500 & 2000 \\
5 & $N_{\text {jet }}$ & $\geq$ & $\mathscr{2}$ & 3 & 4 & 5 & 6 & 7 & 8 & 9 & 10 & 11 \\
\hline 6 & $S_{\mathrm{T}}$ & $>$ & 0 & 0.1 & 0.2 & 0.3 & 0.4 & 0.5 & 0.6 & 0.7 & 0.8 & 0.9 \\
7 & $\Delta \phi_{(J 1)}$ & $>$ & 0 & 0.3 & 0.6 & 0.9 & 1.2 & 1.5 & 1.8 & 2.1 & 2.4 & 2.7 \\
8 & $p_{T(\ell 1)}$ & $>$ & 10 & 15 & 20 & 40 & 60 & 100 & 200 & 500 & 1000 & 2000 \\
9 & $\Delta \phi_{(\ell 1)}$ & $>$ & 0 & 0.3 & 0.6 & 0.9 & 1.2 & 1.5 & 1.8 & 2.1 & 2.4 & 2.7 \\
10 & $M_{T}$ & $>$ & 100 & 100 & 100 & 100 & 100 & 100 & 100 & 100 & 100 & 100 \\
\hline
\end{tabular}

Table 1: Allowed values for each of the cuts described in the text. The preselection cuts (highlighted in bold italic) are shown in the third column (applies to cuts 1 to 5 only). The units of the variables $(1-4,8,10)$ are $\mathrm{GeV}$.

A fraction of the simulated gaugino production events contain high $p_{T}$ jets from initial state parton showers. However the parton shower algorithm is based on the soft and co-linear approximations so this fraction will be smaller than that which would result from higher order matrix element calculations. Our simulation of the trigger can therefore be considered conservative.

Different values of the cuts were applied to events with zero, one, two or three leptons (electrons and muons only) as reconstructed by ATLFAST, as well as to an inclusive lepton ('ptmiss') analysis. In the case of two-lepton events, different cuts were applied according to whether the leptons were of the same sign (SS) or opposite sign (OS).

For each of these analyses the cuts were optimised in the order listed in table 1, and allowed to take one of ten values shown. The significance of the signal was given by $S / \sqrt{B}$ where $S$ and $B$ are the number of signal and background events expected respectively for $100 \mathrm{fb}^{-1}$. For each variable, the cut was chosen to maximise the significance subject to the constraint that $S>10$. The analysis was determined to be successful if significance greater than five was achieved with at least ten events passing for any set of cuts.

This technique will not necessarily generate the global maximum in $S / \sqrt{B}$ since the cut variables are not totally independent, but it is sufficient for the purposes of a large parameter-space scan, and it decreases the chances of over-fitting to a sparse background. 


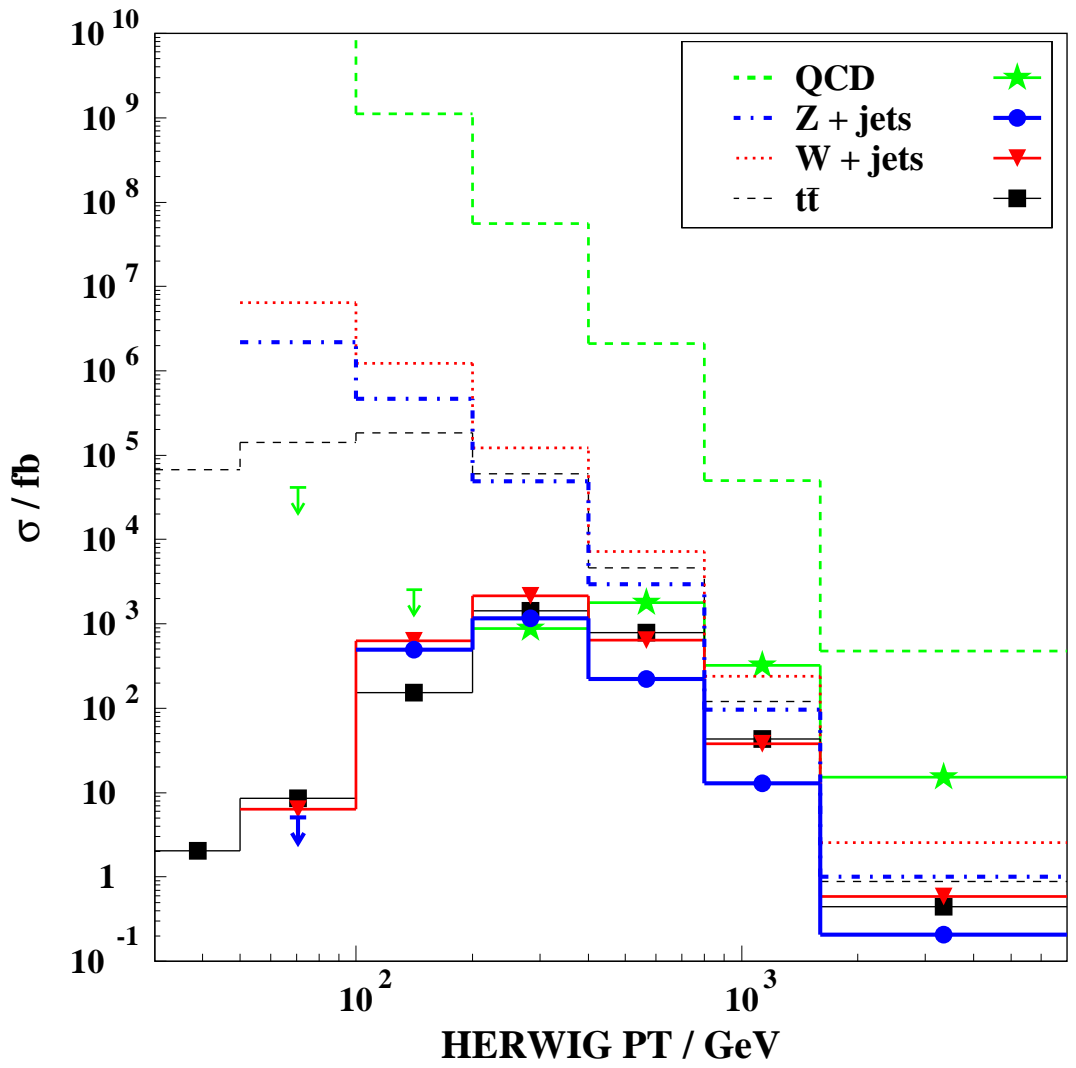

Figure 5: The cross-sections for the $t \bar{t}, W^{ \pm}+$Jets, and $Z^{0}+$ Jets and QCD (excluding $t \bar{t}$ ) backgrounds plotted as a function of the transverse momentum variable which is compared in HERWIG to PTMIN and PTMAX. The production cross-sections are denoted with dashed lines, while the cross-section to pass the preselection cuts are solid lines. Where no events passed the selection cuts (at low PT) arrows indicate 90\% Poisson confidence limits on the selected cross-sections. 


\subsection{Results}

The $5 \sigma$ (and $\geq 10$ event) discovery reaches for $100 \mathrm{fb}^{-1}$ of integrated luminosity in fig. 6 and fig. 7 show that the LHC will be able to distinguish mAMSB from the SM over a large range of parameter space. As expected, the results are similar to previous generic R-parity conserving SUSY searches [22,29,40-42] with reach extending up to squark masses of about $2800 \mathrm{GeV}$, or to gluino masses of about $2100 \mathrm{GeV}$, whichever is the lower.

In general the best reach is obtained in the single-lepton and the inclusive channel. The same-sign dilepton signal is competitive at low $m_{0}$, when $m_{\tilde{\ell}}<m_{\tilde{\chi}_{2}^{0}}-$ a region which includes the model line SPS G - but this sensitivity decreases as $m_{0}$ and $m_{\tilde{\ell}}$ increase. Points with large $m_{0}$ have a spectrum qualitatively similar to that in fig. 2, with heavy squarks and sleptons. Such points can still produce leptons, mostly from heavy chargino or neutralino decays. Another source of leptons is from gluino decays to higgsinos along with a quark and anti-quark from the third generation, followed by leptonic $t$ or $b$ decay.

Along the Snowmass model line ' $\mathrm{G}$ ' $\left(m_{0}=0.0075 \times m_{3 / 2}\right)$ the LHC will be able to measure discrepancies from the Standard Model up to $m_{0}=1050 \mathrm{GeV}, m_{3 / 2}=140 \mathrm{TeV}$, at which point the gluino mass is $2.76 \mathrm{TeV}$ and $m_{\tilde{u}_{L}}=2.70 \mathrm{TeV}$. The Snowmass point SPS 9 lies well within the discovery region for all of the six different analyses. The optimised cuts and the resultant significance are shown in table 2 and 3. The high significance implies that it will be possible to extract further information from the data, for example to determine sparticle masses.

\section{Distinguishing Wino-like LSPs}

Most of the mass-sum rules [14] for mAMSB involve terms like $M_{\tilde{d}_{L}}^{2}-M_{\tilde{u}_{L}}^{2}$ which would be extremely difficult to determine experimentally. The exception is the accidental near-degeneracy of the $\tilde{e}_{L}$ and $\tilde{e}_{R}$, which might be observable at some points, as was noted in sec. 2. However points with large $m_{0}$ have relatively heavy sleptons which would have a small production cross-section at a hadron collider. The Winolike LSP signature of AMSB, and the resultant near-degeneracy of the $\tilde{\chi}_{1}^{+}$and $\tilde{\chi}_{1}^{0}$ will be the robust 'smoking gun' for anomaly mediation, and is applicable beyond the minimal model.

The LHC, like LEP [26], or the Tevatron [15,43] or a future linear $e^{+} e^{-}$collider [44] can use a variety of analyses in the search for long-lived charginos, each tailored to a different regime in $\Delta M_{\tilde{\chi}_{1}}$, according to the $\tilde{\chi}_{1}^{+}$lifetime. The various regimes are ordered by decreasing $\tilde{\chi}_{1}^{+}$lifetime below:

(1) $\Delta M_{\tilde{\chi}_{1}}<m_{\pi^{+}}$. If the pion decay mode is not available, the $\tilde{\chi}_{1}^{+}$will be long lived $(c \tau \gtrsim 1 \mathrm{~m})$ and can leave a track through the muon chambers. Analysis of this type of signature was performed in [45] for a GMSB model with long-lived $\tilde{\tau}_{1}$ 
NLSP, and in [46] for an intermediate scale model with heavy stable leptons. The mass of the chargino can be measured using the muon detector as a timeof-flight system. Additional discrimination can be obtained by considering $\frac{d E}{d x}$ information from the transition radiation tracker. Higher-mass sparticles can then be reconstructed by forming the invariant mass of the stable particle with jets and leptons. Provided the $\tilde{\chi}_{1}^{+}$lifetime, although long, is short enough that a reasonable number of $\tilde{\chi}_{1}^{+}$s decay within the inner detector, the mass difference $\Delta M_{\tilde{\chi}_{1}}$ could also be determined from that fraction using the techniques described below.

(2) $m_{\pi^{+}}<\Delta M_{\tilde{\chi}_{1}} \lesssim 200 \mathrm{MeV}$. This is the regime in which high $p_{T}$ chargino tracks often decay within the body of the inner tracker to soft pions or leptons along with large amounts of missing transverse energy. The details of the detector resolution and track reconstruction algorithm are beyond the scope of this study, but such tracks should provide a striking signature provided they occur in sufficient numbers. The number of $\tilde{\chi}_{1}^{+} \mathrm{s}$ which would be produced at the LHC and decay within a fiducial volume in the active material of the ATLAS tracker is plotted in fig. 8 as a function of $m_{0}$ and $m_{3 / 2}$.

One-prong chargino decays such as $\tilde{\chi}_{1}^{+} \rightarrow \tilde{\chi}_{1}^{0} \pi^{+}$and $\tilde{\chi}_{1}^{+} \rightarrow \tilde{\chi}_{1}^{0} e^{+} \nu_{e}$ will produce 'kinks' at the point where the charged SM particle is softly emitted. In some cases the SM particle will go undetected and the high $p_{T}$ chargino track will seem to disappear. Such decays, producing track stubs, could also be detected with a dedicated off-line analysis.

Even though rather strict triggering requirements have been made, there are at least ten such events for $100 \mathrm{fb}^{-1}$ over almost the entire plotted parameter-space provided $m_{3 / 2} \lesssim 185 \mathrm{TeV}$. This signature should be essentially free of physics background, so 'detector' backgrounds will dominate. Since ATLAS should have three barrel pixel layers with $r<122 \mathrm{~mm}$ with noise occupancy of less than $10^{-5}$, and average physics occupancy of the order of $10^{-4}$ the instrumental backgrounds should also be under control $[47,48]$. Such a search could therefore achieve a large reach and would be sufficient to identify a near-degenerate LSP model.

(3) $200 \mathrm{MeV} \lesssim \Delta M_{\tilde{\chi}_{1}} \lesssim$ a few GeV. Chargino decays which occur before the first tracking layer can still be useful provided that the soft track from the SM particle can be found, using e.g. electron identification, track impact parameter, isolation from other tracks, and the direction of the $\boldsymbol{p}_{T} 2$-vector.

The maximum value of $\Delta M_{\tilde{\chi}_{1}}$ in minimal AMSB with $\tan \beta=10$ is less than about $200 \mathrm{MeV}$ except close to the region of no electroweak symmetry breaking. This leads to a decay distance $c \tau \gtrsim$ a few centimeters, and corresponds to category (2) 
above. However, other models such as 0-II string models [30,31,49] have Wino-like $\tilde{\chi}_{1}^{+}$ and $\tilde{\chi}_{1}^{0}$ with $\Delta M_{\tilde{\chi}_{1}}$ typically of the order of a GeV. Such models fall into category (3), which is potentially the most difficult at a hadron collider because the short-lived $\tilde{\chi}_{1}^{+}$s will not be directly observable, and their softly emitted SM daughters suffer from a large background from other low-momentum tracks. In sec. 5.1 we define a range of model points which allows us to study this regime of shorter lifetimes. Some of the methods which can enable the LHC to probe this region are explored in sec. 5.2 .

\subsection{Definition of points}

The tree-level neutralino mass matrix is:

$$
\left(\begin{array}{cccc}
M_{1} & 0 & -m_{Z} c_{\beta} s_{W} & m_{Z} s_{\beta} s_{W} \\
0 & M_{2} & m_{Z} c_{\beta} c_{W} & -m_{Z} s_{\beta} c_{W} \\
-m_{Z} c_{\beta} s_{W} & m_{Z} c_{\beta} c_{W} & 0 & -\mu \\
m_{Z} s_{\beta} s_{W} & m_{Z} s_{\beta} c_{W} & -\mu & 0 \\
& & &
\end{array}\right)
$$

and the tree-level chargino mass matrix is:

$$
\left(\begin{array}{cc}
M_{2} & \sqrt{2} m_{W} s_{\beta} \\
\sqrt{2} m_{W} c_{\beta} & \mu
\end{array}\right)
$$

where $s_{W} \equiv \sin \theta_{W}, c_{W} \equiv \cos \theta_{W}, s_{\beta} \equiv \sin \beta, c_{\beta} \equiv \cos \beta$ and we use the conventions of [50]. The mass difference $m_{\tilde{\chi}_{1}^{+}}-m_{\tilde{\chi}_{1}^{+}} \equiv \Delta M_{\tilde{\chi}_{1}}$ is highly suppressed at tree-level, so the leading 1-loop correction can be important. It takes the form [51]:

$$
\Delta M_{\tilde{\chi}_{1}}{ }^{(1-\text { loop })}=\frac{\alpha_{2} M_{2}}{4 \pi}\left[F\left(\frac{m_{W}}{M_{2}}\right)-c_{W}^{2} F\left(\frac{m_{Z}}{M_{2}}\right)+5 s_{W}^{2}\right],
$$

where $F(a) \equiv \int_{0}^{1} d x(2+2 x) \log \left[x^{2}+(1-x) a^{2}\right]$, and is included in ISAJET.

We are interested in the collider phenomenology of long-lived charginos with various lifetimes. From (5.1) and (5.2), the leading tree-level mass difference term [51] $\Delta M_{\tilde{\chi}_{1}} \propto 1 / \mu^{2}$. In order to explore different regimes in $\Delta M_{\tilde{\chi}_{1}}$, we define new points based on SPS 9 and another minimal anomaly-mediated point with $m_{0}=500 \mathrm{GeV}$, $m_{3 / 2}=36 \mathrm{TeV}, \mu>0$ and $\tan \beta=10$. We then decreased the value of the $\mu$ parameter at the electroweak scale to the values shown in table 4 .

In order to maintain consistent electroweak symmetry breaking when decreasing $\mu$, the Higgs soft mass parameters, $m_{H_{1}}^{2}$ and $m_{H_{2}}^{2}$, must allowed to vary, as can be seen from tree-level equation,

$$
\mu^{2}=\frac{m_{H_{1}}^{2}-m_{H_{2}}^{2} \tan ^{2} \beta}{\tan ^{2} \beta-1}-\frac{1}{2} m_{Z}^{2} .
$$

The points with adjusted $\mu$ are then no longer in the minimal anomaly-mediated scenario, since $m_{H_{1}}^{2}$ and $m_{H_{2}}^{2}$ are not those which would be predicted from the mAMSB 


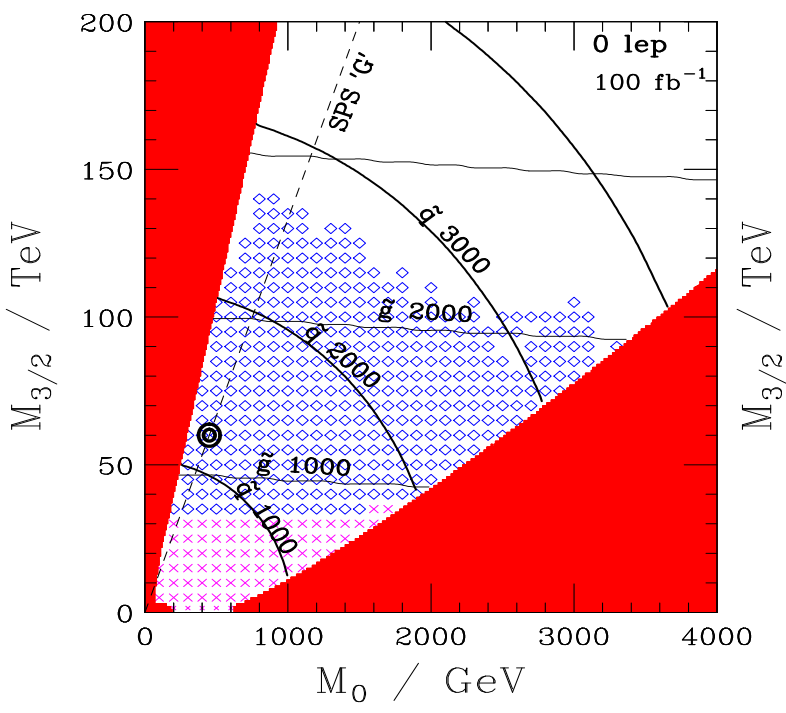

(a)

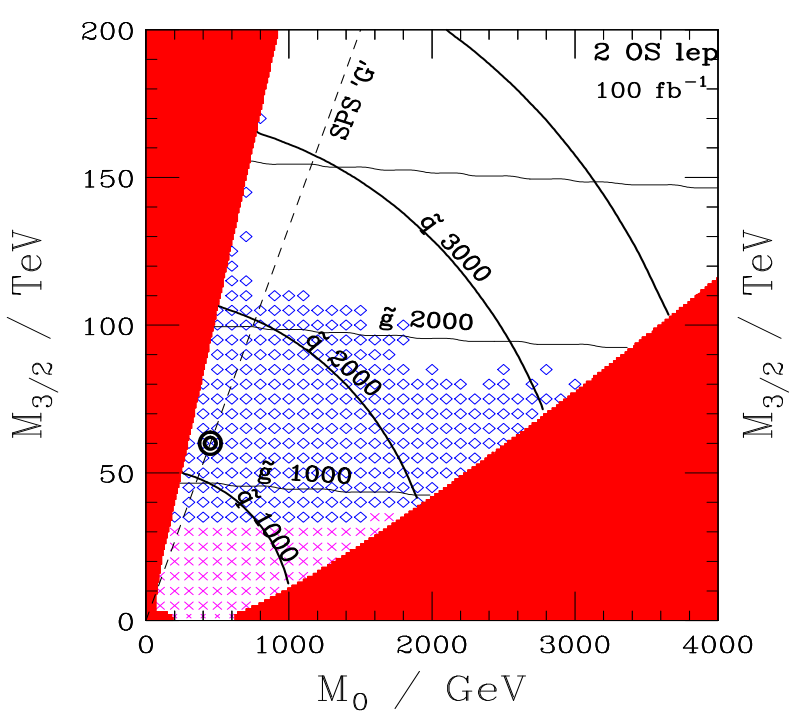

(c)

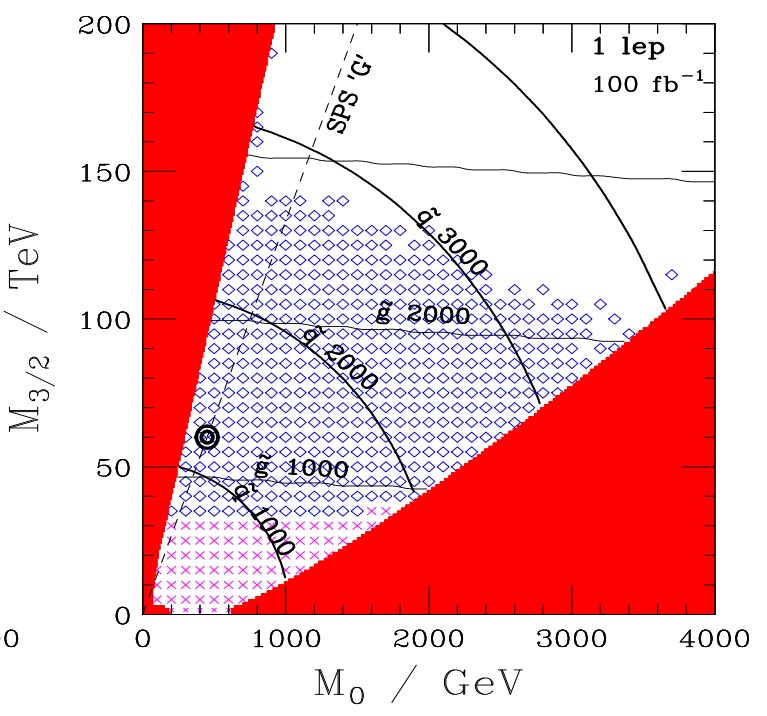

(b)

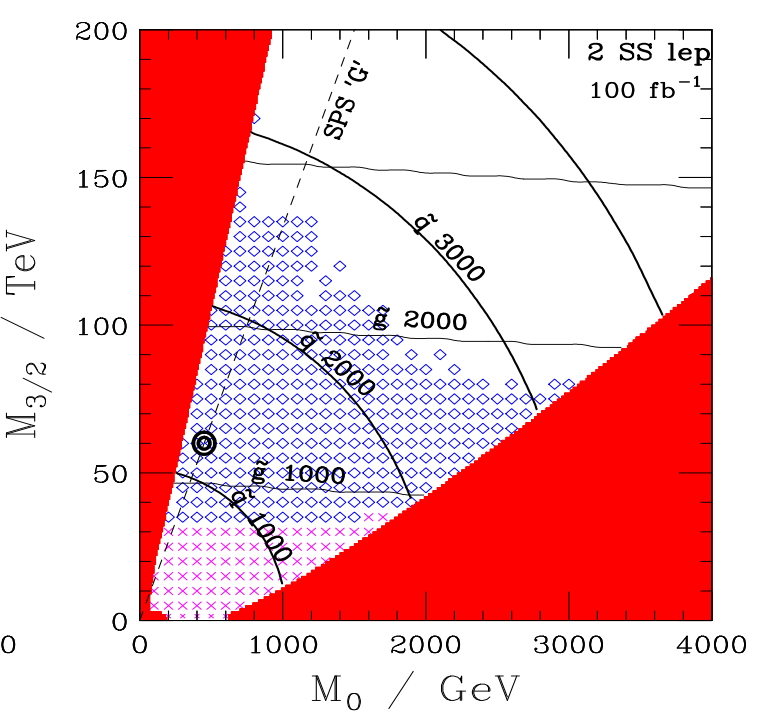

(d)

Figure 6: The $5 \sigma$ (and $\geq 10$ event) discovery region for mAMSB in the $m_{0}-m_{3 / 2}$ plane, is shown by the light blue diamonds, for the (a) no lepton, (b) single lepton, (c) two opposite-sign leptons, (d) two same-sign leptons channels. The other parameters are $\tan \beta=10$ and $\mu>0$ in all cases. The solid red regions are excluded because of lack of electroweak symmetry breaking (bottom right) or because of charge- or colour-breaking minima or non- $\tilde{\chi}_{1}^{0}$ LSP (left). The low $m_{3 / 2}$ region excluded by the LEP limit [26] on the chargino mass is indicated by purple crosses. The Snowmass mAMSB point SPS 9 at $m_{0}=450 \mathrm{GeV}, m_{3 / 2}=60 \mathrm{TeV}$ is marked with a pair of concentric circles, through which passes the Snowmass slope 'model line G' (dashed line). Contours of $\tilde{u}_{L}$ and $\tilde{g}$ iso-mass are shown as solid lines. 


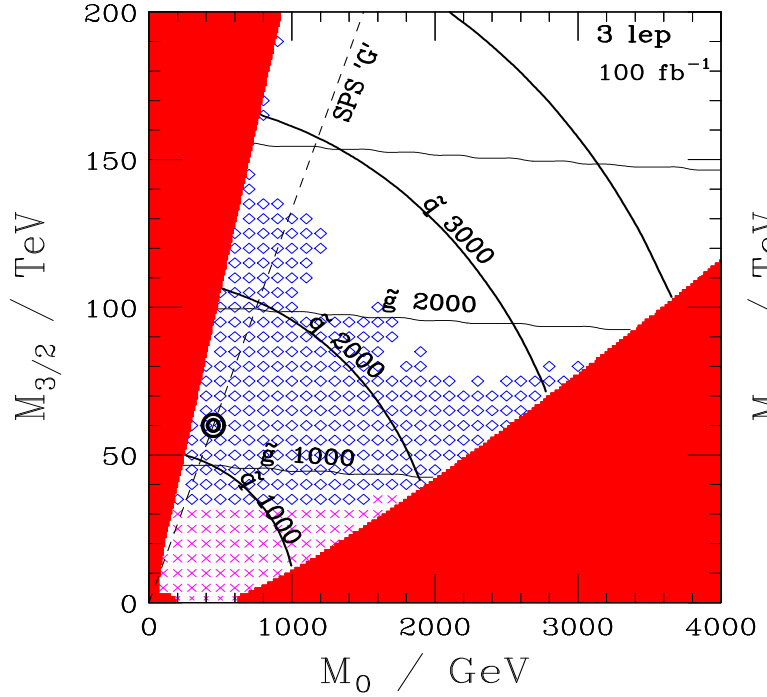

(a)

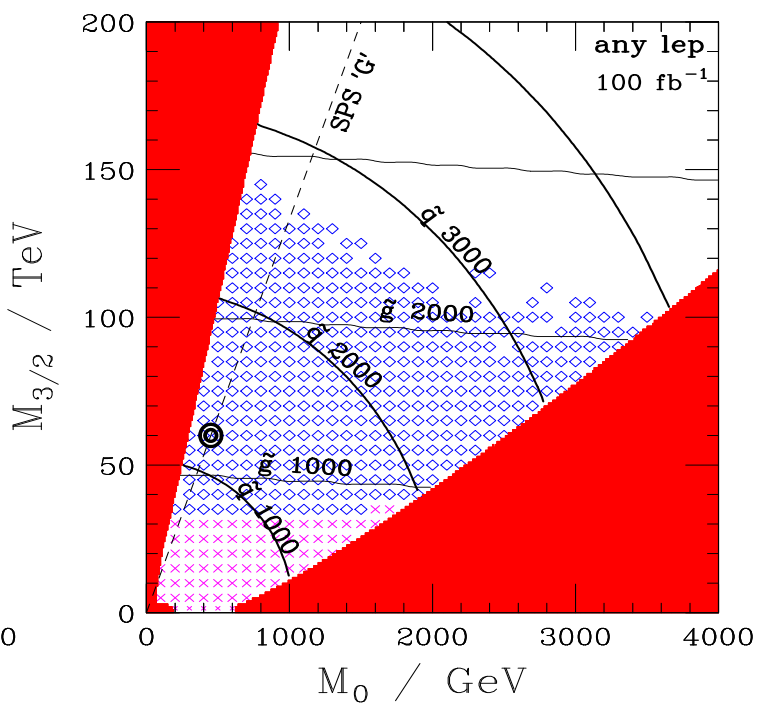

(b)

Figure 7: As fig. 6 but for the (a) the three lepton channel and (b) the inclusive ('missing pt') channel.

\begin{tabular}{|l|c|c|c|c|c|c|c|c|c|}
\hline Analysis & $\mathscr{E}_{T}$ & $p_{T(J 1)}$ & $p_{T(J 2)}$ & $\sum p_{T}$ & $N_{\text {jet }}$ & $S_{\mathrm{T}}$ & $\Delta \phi_{(J 1)}$ & $p_{T(\ell 1)}$ & $\Delta \phi_{(\ell 1)}$ \\
\hline ptmiss & 600 & 100 & 200 & 1000 & 9 & 0.2 & 0.3 & N/A & N/A \\
0 Lep & 800 & 150 & 200 & 1000 & 10 & 0.1 & 0 & N/A & N/A \\
1 Lep & 500 & 200 & 200 & 200 & 10 & 0.6 & 0 & 10 & 0 \\
2 Lep OS & 800 & 100 & 150 & 200 & 9 & 0.7 & 0 & 10 & 0 \\
2 Lep SS & 500 & 100 & 600 & 200 & 2 & 0.1 & 0 & 10 & 0 \\
3 Lep & 400 & 400 & 200 & 200 & 9 & 0 & 0 & 10 & 0 \\
\hline
\end{tabular}

Table 2: The value of the cuts which maximised the significance $(S / \sqrt{B})$ for each of the analyses at the Snowmass mAMSB Point SPS 9 for $\int \mathcal{L}=100 \mathrm{fb}^{-1}$. The $M_{T}$ cut applied only to the single lepton channel and was kept at $100 \mathrm{GeV}$. 


\begin{tabular}{|l||c|c|c||c|c|c|}
\hline \multicolumn{1}{|c||}{} & \multicolumn{3}{c||}{ SPS 9 } & \multicolumn{3}{c|}{ Point d'Aix } \\
\hline Analysis & $S$ & $B$ & $S / \sqrt{B}$ & $S$ & $B$ & $S / \sqrt{B}$ \\
\hline \hline ptmiss & 3300 & 151 & 270 & 2650 & 0.25 & 5200 \\
0 Lep & 950 & 21 & 206 & 7800 & 4.46 & 3700 \\
1 Lep & 151 & 0.00005 & 21000 & 930 & 0.00005 & $10^{5}$ \\
2 Lep OS & 15 & 0.00005 & 2100 & 62 & 0.00005 & 8800 \\
2 Lep SS & 13 & 0.0005 & 1900 & 67 & 0.0048 & 970 \\
3 Lep & 206 & 0.0001 & 20400 & 19 & 0.00010 & 1900 \\
\hline
\end{tabular}

Table 3: The expected number of signal $(S)$, and background $(B)$ events and the significance $(S / \sqrt{B})$ for for each of the analyses, at the Snowmass mAMSB Point SPS 9 and the Point d'Aix, for $\int \mathcal{L}=100 \mathrm{fb}^{-1}$. For all six analyses and for both points the backgrounds are dominated by high $p_{T}$ vector boson production in association with jets.

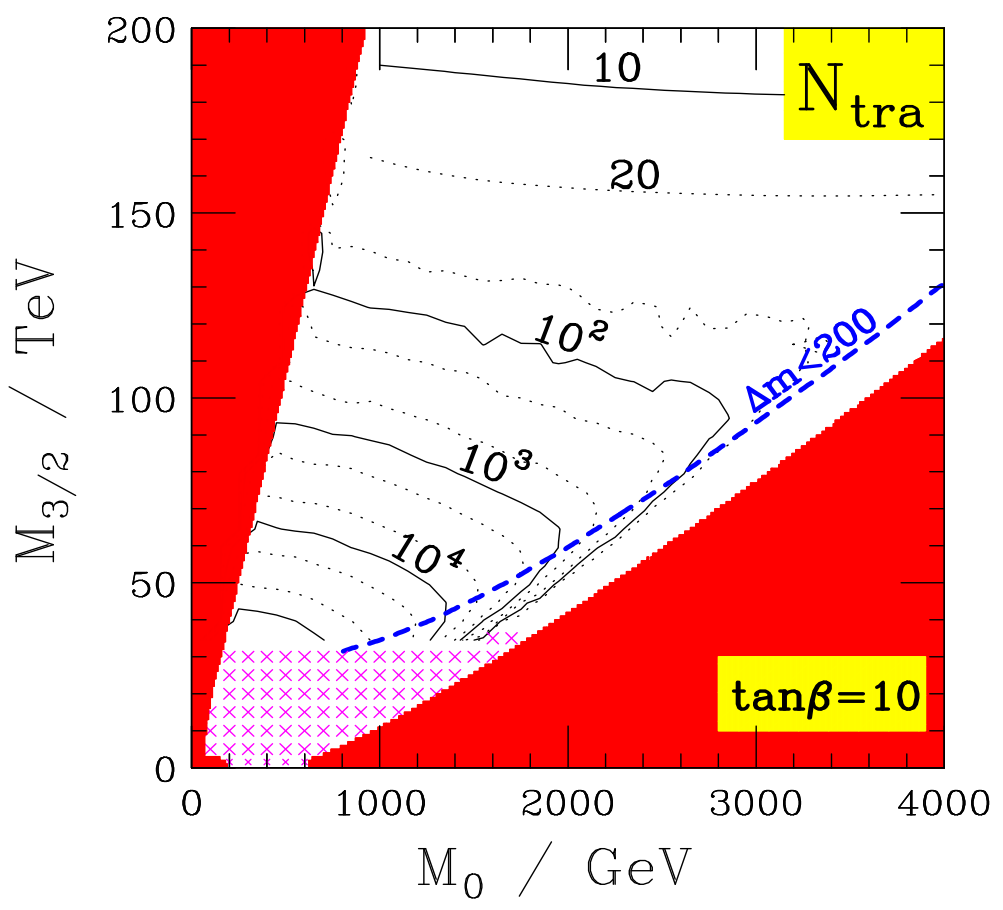

Figure 8: The number of $\tilde{\chi}_{1}^{ \pm} \rightarrow \tilde{\chi}_{1}^{0}$ decays expected within the central region of the detector $(|\eta|<2)$ with transverse decay vertices between $100 \mathrm{~mm}$ and $800 \mathrm{~mm}$ from the interaction point for integrated luminosity of $100 \mathrm{fb}^{-1}$. The initial track from the chargino is required have $p_{T}>10 \mathrm{GeV}$. All events are required to have passed the preselection cuts (table 11) which ensure that the event will be triggered. The 10 event and 20 event contours have been smoothed for clarity. $\Delta M_{\tilde{\chi}_{1}}$ is less than $200 \mathrm{MeV}$ above and to the left of the dashed blue line on the bottom right-hand side of the plot. 


\begin{tabular}{|l|c|c|c|c|c|c|}
\hline Point & $\mu$ & $\begin{array}{c}m_{\tilde{\chi}_{1}^{+}} \\
(\mathrm{GeV})\end{array}$ & $\begin{array}{c}\Delta M_{\tilde{\chi}_{1}} \\
(\mathrm{MeV})\end{array}$ & $\begin{array}{c}c \tau \\
(\mathrm{mm})\end{array}$ & $\tilde{\chi}_{1}^{+} \rightarrow \tilde{\chi}_{1}^{0} e^{+} \nu_{e}$ & $\tilde{\chi}_{1}^{+} \rightarrow \tilde{\chi}_{1}^{0} \mu^{+} \nu_{\mu}$ \\
\hline SPS 9 & 864 & 171 & 164 & 56 & $2.0 \%$ & $0.2 \%$ \\
SPS-300 & 300 & 165 & 886 & 0.11 & $17.0 \%$ & $15.9 \%$ \\
SPS-250 & 250 & 159 & 1798 & 0.004 & $21.9 \%$ & $21.5 \%$ \\
\hline A & 533 & 107 & 181 & 34 & $2.0 \%$ & $0.3 \%$ \\
A-250 & 250 & 101 & 766 & 0.20 & $15.4 \%$ & $13.9 \%$ \\
A-200 & 200 & 97 & 1603 & 0.007 & $22.5 \%$ & $22.2 \%$ \\
\hline
\end{tabular}

Table 4: The mass of the lightest chargino, its mass difference with the LSP, decay length and leptonic branching ratios for six points. SPS 9 is the Snowmass mAMSB point, from which we define SPS 300, and SPS 250 by adjusting $\mu$ at the electroweak scale to produce different $\Delta M_{\tilde{\chi}_{1}}$. Point $\mathbf{A}$ is the mAMSB point with $m_{0}=500 \mathrm{GeV}, m_{3 / 2}=36 \mathrm{TeV}, \mu>0$ and $\tan \beta=10$, from which $\mu$ is adjusted at the electroweak scale to produce $\mathbf{A - 2 5 0}$ and A-200.

parameters $m_{0}$ and $m_{3 / 2}$. Decreasing $\mu$ has the side-effect of decreasing the masses of the higgsinos, and can change the phenomenology somewhat - for example by opening chains such as $\tilde{q} \rightarrow \tilde{\chi}_{2}^{+} q \rightarrow \tilde{\chi}_{1}^{+} q Z^{0}$. When followed by leptonic $Z^{0}$ decay these chains could, in principle, be used to further constrain the dynamics. However the only requirement we make for our analysis is that the decay $\tilde{q} \rightarrow \tilde{\chi}_{1}^{+} q$ occurs, producing highly boosted $\tilde{\chi}_{1}^{ \pm}$pairs in association with jets.

\subsection{Identifying chargino decay products}

In this section we demonstrate how the low- $p_{T}$ tracks from the charged SM daughters of $\tilde{\chi}_{1}^{+}$decays can be identified and used to constrain $\Delta M_{\tilde{\chi}_{1}}$. To do so we must distinguish these tracks from the large number of other low- $p_{T}$ tracks from the particles generated in the proton remnant interactions, and from any pile-up events. To avoid having to deal with the contamination from multiple pile-up, in this section we simulate the initial three years of 'low luminosity' $\left(10^{34} \mathrm{~cm}^{-2} \mathrm{~s}^{-1}\right)$ running of the LHC, which is expected to provide $30 \mathrm{fb}^{-1}$ of integrated luminosity with an average of one inelastic collision per bunch-crossing. The loss of resolution from pile-up is therefore not simulated in this section.

The HERWIG soft underlying event model, based on the UA5 collaboration [52] $p \bar{p}$ Monte-Carlo, is known to underestimate the number of tracks with $p_{T}>1 \mathrm{GeV}$ [53], because it does not model the semi-hard physics of multiple parton interactions. To ensure that our analysis was insensitive to contamination from such tracks, a sample of 5000 inclusive QCD events with PTMIN $=4.5 \mathrm{GeV}$ was generated. We refer to these as 'minimum bias events' because they simulate (better than the default HERWIG soft minimum bias option) the expected minimum bias distribution of tracks, including a tail at higher $p_{T}$ due to the onset of hard scattering. The particles from this minimum 


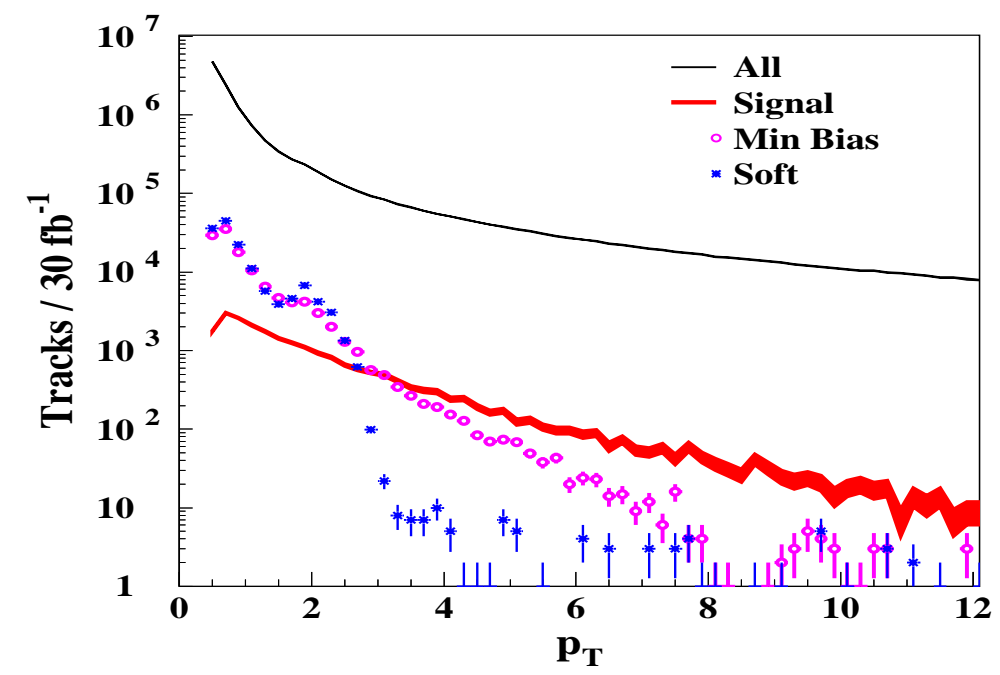

Figure 9: The transverse momentum distribution of reconstructed charged tracks for the point SPS-250 (defined in table (4) after selecting events with $\mathbb{E}_{T}>200 \mathrm{GeV}$. The thin black line shows all tracks; the distribution for particles originated from $\tilde{\chi}_{1}^{ \pm}$decays (the signal) is highlighted by the thick red line, where the thickness shows the statistical uncertainty; those from the minimum bias event and from the soft underlying event are denoted by circles and asterisks respectively.

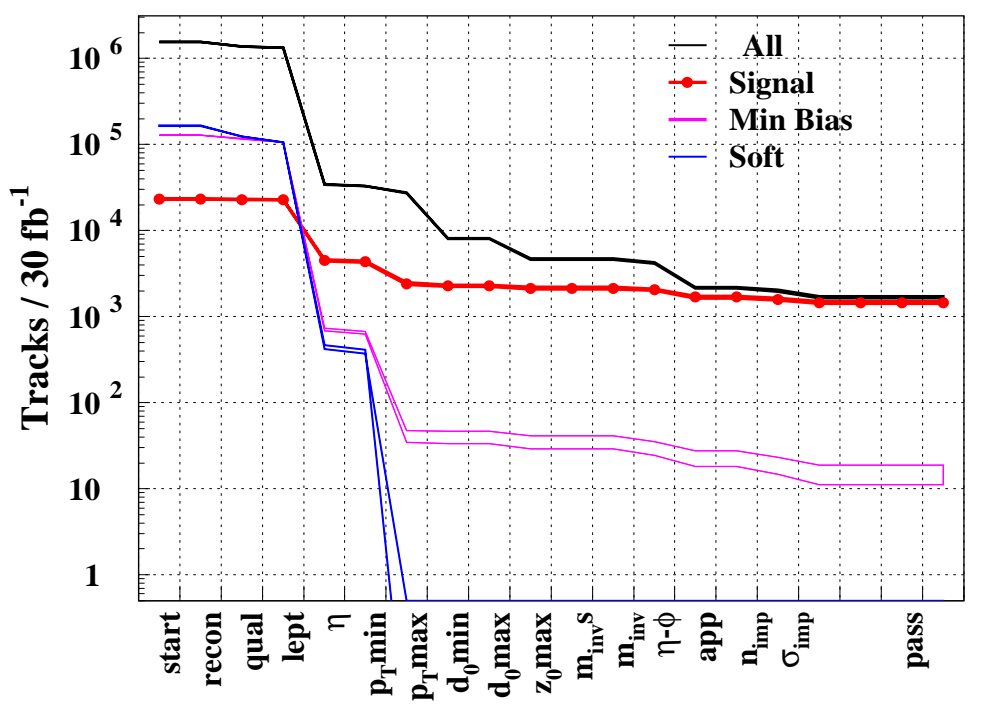

Figure 10: The number of tracks surviving subsequent cuts, after a preselection cut $\mathbb{E}_{T}>200 \mathrm{GeV}$ for the point SPS-250 (defined in table 4 ). The signal tracks (from $\tilde{\chi}_{1}^{ \pm}$ decays) are also plotted separately, as are tracks from the HERWIG soft underlying event and the additional minimum bias QCD event. Not all cuts are used in any particular analysis. For this point the most effective cuts are those on the $p_{T}$ of the track, and on selecting leptons ('lept'), while further cuts on the transverse impact parameter $\left(d_{0}\right)$ and isolation $(\eta-\phi)$ improve the selection. 
bias event were added to the SUSY event in addition to HERWIG's usual underlying event.

An initial selection requiring a high $p_{T}$ jet and large missing transverse energy was made to trigger the event, reduce the SM background, and select those SUSY events in which the neutralino was highly boosted. In this section we are dealing with the short-lifetime regime $(c \tau<1 \mathrm{~mm})$, so this boost will almost certainly not be sufficient to allow the chargino to live long enough to decay in the body of the detector. However a boosted chargino means that even particles which are very softly emitted in the chargino rest frame will have a transverse momentum

$$
p_{T x} \sim \frac{p_{T \tilde{\chi}_{1}^{0}}}{m_{\tilde{\chi}_{1}^{0}}} \times m_{x}
$$

as measured in the detector, where $x$ is the charged SM daughter particle and $p_{T \tilde{\chi}_{1}^{0}}$ is of the order of the missing transverse momentum. This extra $p_{T}$ assists the reconstruction of the SM daughters of charginos and helps distinguish them from even softer particles coming from the underlying event (fig. 9).

The tracks from SM daughters of chargino decays have other properties which can help in their identification. For larger $\Delta M_{\tilde{\chi}_{1}}$ the $\tilde{\chi}_{1}^{+}$branching ratio to each of muon

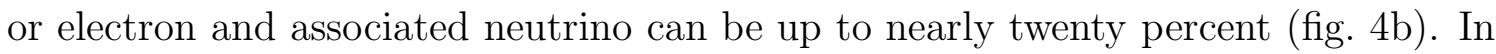
the ATLAS experiment electrons and muons will be distinguished from other tracks by transistion radiation and by penetration respectively (see appendix B).

Much of the background is associated with heavy-quark decay and can be removed by applying isolation cuts (table 5) which require that for any track, $i$, to be a candidate:

- no other track is found with $\Delta R_{i j}=\sqrt{\left(\Delta \eta_{i j}\right)^{2}+\left(\Delta \phi_{i j}\right)^{2}}$ less than some value $\Delta R_{i j}^{\min }$

- the invariant mass of the track with another track, $j$, is greater than $M_{i j}^{\min }$ for all $j$, where it is assumed that $m_{i}=m_{j}=m_{\pi^{+}}$;

- no other track with $R_{i j}<R_{\text {imp }}$ has $d_{0}^{(j)} / \sigma\left(d_{0}^{(j)}\right)>3$, where $d_{0}^{(j)}$ is the transverse impact parameter, and $\sigma\left(d_{0}^{(j)}\right)$ is its uncertainty;

- $\sum_{j} d_{0}^{(j)} / \sigma\left(d_{0}^{(j)}\right)<\sigma_{\text {imp }}$ where the sum is over all $j \neq i$ with $R_{i j}<R_{\text {imp }}$.

\subsection{Results}

We selected those events in which precisely two tracks satisfied the track cuts listed in table 5. The numbers of tracks from chargino decays and from other sources which passed subsequent cuts are shown in fig. 10 for the point SPS-250. We then considered the Cambridge $m_{T X}$ variable which (as described in appendix A) remains sensitive to $\Delta M_{\tilde{\chi}_{1}}$ even when there are uncertainties in the neutralino mass or the 


\begin{tabular}{|c|c|c|c|}
\hline & Points & SPS-300，A-250 & SPS-250, A-200 \\
\hline \multirow[t]{3}{*}{ Event: } & $\mathbb{E}_{T}^{\min }$ & 500 & 500 \\
\hline & $p_{T(J 1)}^{\min }$ & 400 & 400 \\
\hline & $S_{T}^{\min }$ & 0.05 & 0.05 \\
\hline \multirow[t]{10}{*}{ Track: } & $p_{T}^{\min }$ & 2.0 & 2.0 \\
\hline & $p_{T}^{\max }$ & 5 & 10 \\
\hline & $d_{0}^{\min }$ & 0.03 & 0 \\
\hline & $d_{0}^{\max }$ & 0.3 & 0.1 \\
\hline & $|\eta|^{\max }$ & 1.5 & 2 \\
\hline & $\Delta R_{i j}^{\min }$ & 0.45 & 0.2 \\
\hline & $M_{i j}^{\min }$ & 0.9 & 0.4 \\
\hline & $R_{\mathrm{imp}}$ & 0.4 & 0.4 \\
\hline & $\sigma_{\mathrm{imp}}$ & 3.0 & 3.0 \\
\hline & Particle & any & $\ell \in e, \mu$ \\
\hline
\end{tabular}

Table 5: The cuts applied to events and to tracks for the different points in table 4 . The missing transverse energy $\left(\mathbb{F}_{T}\right)$, leading jet transverse momentum $\left(p_{T(J 1)}\right)$, and transverse sphericity $\left(S_{T}\right)$ cuts were applied to the whole event, while the other cuts were applied track by track. The track transverse momentum was required to lie in the range $p_{T}^{\min } \rightarrow p_{T}^{\max }$; its transverse impact parameter in the range $d_{0}^{\min } \rightarrow d_{0}^{\max }$; and the absolute value of its pseudorapidity was required to be less than $|\eta|^{\text {max }}$. The other cuts are described in the text. Energy, momentum and mass units are $\mathrm{GeV}$; the impact parameter, $d_{0}$, is measured in $\mathrm{mm}$. 


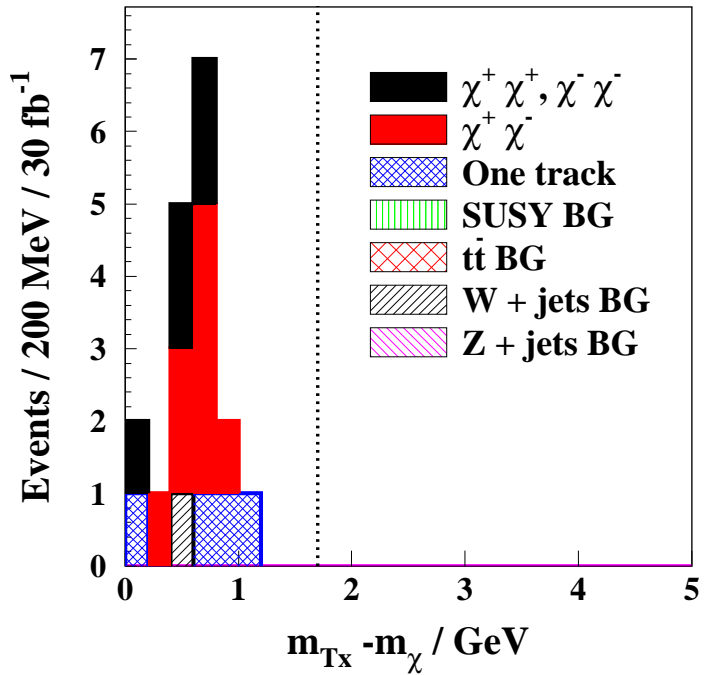

(a)

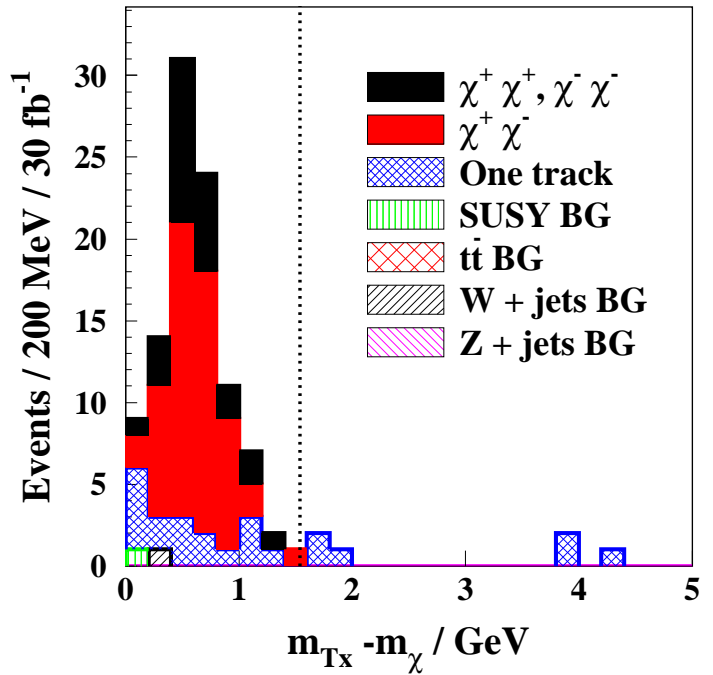

(b)

Figure 11: The $m_{T 4}-m_{\tilde{\chi}_{1}^{0}}$ distribution for the points (a) SPS-250, and (b) A-200

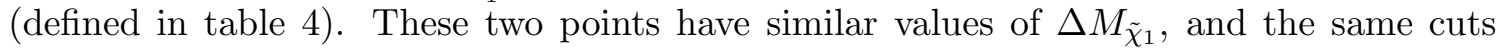
were applied to both. Only events in which exactly two leptons passed the selection cuts are plotted. The signal tracks are those from $\tilde{\chi}_{1}^{+}$and $\tilde{\chi}_{1}^{-}$decays. Events where only one of the two tracks has been successfully identified are plotted separately. The vertical dotted line indicates $\Delta M_{\tilde{\chi}_{1}}=m_{\tilde{\chi}_{1}^{+}}-m_{\tilde{\chi}_{1}^{0}}$.

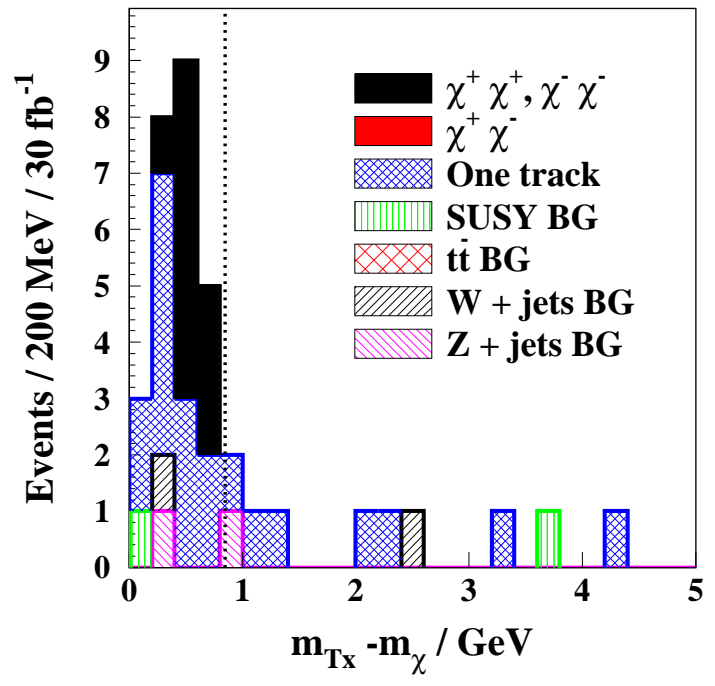

(a)

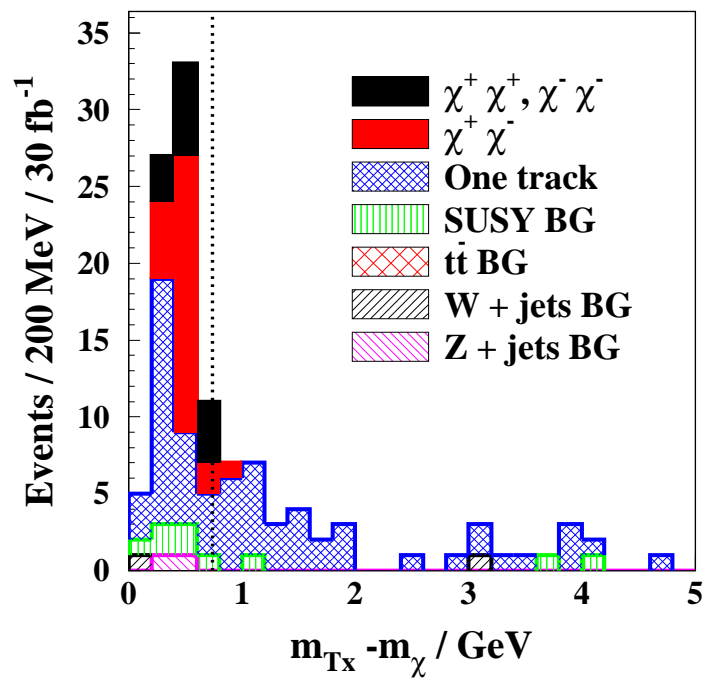

(b)

Figure 12: The $m_{T 2}-m_{\tilde{\chi}_{1}^{0}}$ distribution for (a) the point SPS-300, and (b) the point A-250. These two points have similar values of $\Delta M_{\tilde{\chi}_{1}}$, and the same cuts were applied to both. Only events in which exactly two particles (of any type) passed the selection cuts are plotted. The peak is closer than in fig. 11 to the upper kinematic limit at $m_{T 2}-m_{\tilde{\chi}_{1}^{0}}=$ $\Delta M_{\tilde{\chi}_{1}}$ (dotted line) since there are only two missing particles - the two neutralinos. 
missing transverse momentum, providing an important handle on Wino-LSP physics at hadron colliders. We assume that both the squark mass scale, $m_{\tilde{q}}$, and the $\tilde{\chi}_{1}^{0}$ mass have been previously determined from the measurement of other kinematic edges as described in $[22-24,42]$, and plot distributions of $m_{T X}-m_{\tilde{\chi}_{1}^{0}}$ which has the property:

$$
0<\left(m_{T X}-m_{\tilde{\chi}_{1}^{0}}\right) \leq\left(m_{\tilde{\chi}_{1}^{+}}-m_{\tilde{\chi}_{1}^{0}}\right) \equiv \Delta M_{\tilde{\chi}_{1}} .
$$

For the two points with $\Delta M_{\tilde{\chi}_{1}} \approx 1.7 \mathrm{GeV}$, we selected isolated leptons and plotted distributions of $m_{T 4}-m_{\tilde{\chi}_{1}^{0}}$. As can be seen in fig. 11, A-200 has more signal events passing the cuts than SPS-250, but the peak position is the same in both cases.

As $\Delta M_{\tilde{\chi}_{1}}$ decreases, the leptonic branching ratios decrease (see fig. $4 \mathrm{~b}$ ) and the detector's ability to distinguish the lower $p_{T}$ leptons from hadrons is diminished (see appendix $\mathrm{B}$ ). For this reason, at the two points with $\Delta M_{\tilde{\chi}_{1}} \approx 800 \mathrm{MeV}$ we select events with two tracks of any type satisfying the cuts in the third column of table 5 . Most of these tracks will be pions because of the large branching ratio to $\pi^{ \pm}$or $\pi^{ \pm} \pi^{0}$. The $m_{T 2}-m_{\tilde{\chi}_{1}^{0}}$ distributions for these two points are plotted in fig. 12.

For all four points the narrow peaks indicate that $\Delta M_{\tilde{\chi}_{1}}$ is of the order of $1 \mathrm{GeV}$. This confirms the Wino-like nature of the LSP, providing the 'smoking gun' signature for anomaly mediation.

Each $m_{T X}$ distribution depends principally on $\Delta M_{\tilde{\chi}_{1}}$, on the neutralino mass and the momentum distribution of the charginos. The latter depends largely on $m_{\tilde{q}}$ so if both $m_{\tilde{q}}$ and the lightest neutralino mass were already measured, then $\Delta M_{\tilde{\chi}_{1}}$ can be measured by fitting to each $m_{T X}-m_{\tilde{\chi}_{1}^{0}}$ distribution.

To demonstrate that $m_{T 4}$ can indeed make a quantitative measurement of $\Delta M_{\tilde{\chi}_{1}}$, a phase-space Monte-Carlo program was used to generate very simple 'events' in which pairs of squarks decayed via the chain $\tilde{q} \rightarrow \tilde{\chi}_{1}^{+} q \rightarrow \tilde{\chi}_{1}^{0}$ e $\nu_{e} q \cdot m_{T 4}-m_{\tilde{\chi}_{1}^{0}}$ distributions were produced for those events in which $\not p_{T}>500 \mathrm{GeV}$, and the peak determined from a gaussian fit. The correlation between the fitted peak positions and the input values of $\Delta M_{\tilde{\chi}_{1}}$ is shown in fig. 13. The peak of the $m_{T 4}-m_{\tilde{\chi}_{1}^{0}}$ distribution for point A-200 (fig. 11 b), was likewise determined from a gaussian fit. As can be seen from fig. 13 the mass difference $\Delta M_{\tilde{\chi}_{1}}$ can be measured at that point with a statistical uncertainty of approximately $150 \mathrm{MeV}(10 \%)$.

\section{Other constraints}

\subsection{Cosmological relic density}

If R-Parity is conserved then the lightest supersymmetric particle can be a good candidate for the cold (non-relativistic) dark matter hypothesised by astrophysicists and cosmologists. The cold dark matter contribution to critical density of the universe 


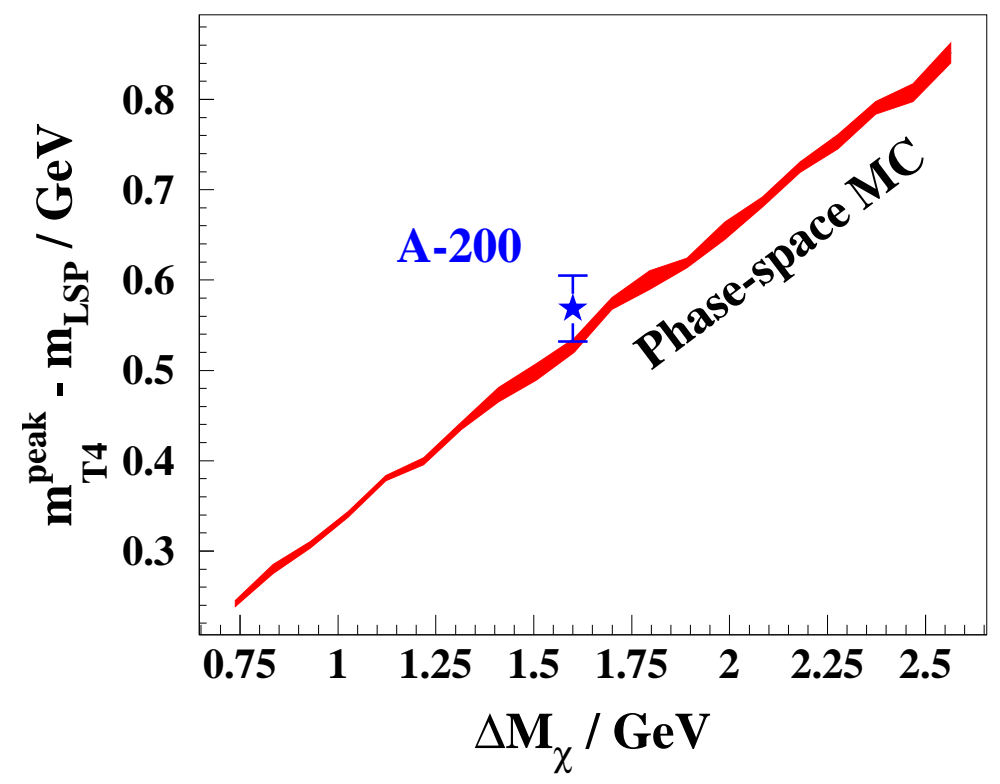

Figure 13: The diagonal line shows how the $m_{T 4}-m_{\tilde{\chi}_{1}^{0}}$ peak value depends on $\Delta M_{\tilde{\chi}_{1}}$. The thickness of the line indicates the uncertainty in the peak position from a gaussian fit to the distribution combined in quadrature with a $10 \%$ uncertainty in the LSP mass and a $10 \%$ uncertainty in the squark mass scale. The peak of the $m_{T 4}-m_{\tilde{\chi}_{1}^{0}}$ distribution for the point A-200 is marked with a star at its input value of $\Delta M_{\tilde{\chi}_{1}}$; the error bar shows its uncertainty.

provided by the LSP is given by:

$$
\omega_{\mathrm{CDM}} \equiv \Omega_{\mathrm{CDM}} h^{2}=\frac{m_{\tilde{\chi}_{1}^{0}} n_{\tilde{\chi}_{1}^{0}}}{\rho_{\text {crit }} / h^{2}}
$$

where $\rho_{\text {crit }}=3 H^{2} / 8 \pi G$ is the critical density, $n_{\tilde{\chi}_{1}^{0}}$ is the number density of LSPs, and $H=h \times 100 \mathrm{~km} \mathrm{~s}^{-1} \mathrm{Mpc}^{-1}$ is the Hubble constant.

Astronomical estimates from measurements of the acoustic power spectrum of the cosmic microwave background anisotropy [54-58] suggest that $\omega_{\mathrm{CDM}}=0.106 \pm 0.010$. Further constraints from the $2 \mathrm{dF}$ Galactic Redshift Survey [59] lead to $\omega_{\mathrm{CDM}}=$ $0.1151 \pm 0.0091$, assuming a flat universe $\left(\Sigma_{i} \Omega_{\mathrm{i}}=1\right)$.

In AMSB the Wino-like $\tilde{\chi}_{1}^{0}$ and $\tilde{\chi}_{1}^{+}$undergo rapid annihilation though reactions such as $\tilde{\chi}_{1}^{0} \tilde{\chi}_{1}^{0} \rightarrow W^{+} W^{-}$and $\tilde{\chi}_{1}^{0} \tilde{\chi}_{1}^{+} \rightarrow q q^{\prime}$. The relic density of cold dark matter was calculated for AMSB using the program micrOmegas [60], which includes the annihilation channels listed in table 6. The results, in fig. 14, show that the value of $\omega_{\mathrm{CDM}}$ is small throughout the region, and about a factor of two less than was 
estimated in [4]:

$$
\omega_{\mathrm{CDM}}^{\mathrm{no}-\text { coanh }} \approx 5 \times 10^{-4} \times\left(\frac{m_{\tilde{\chi}_{1}^{0}}}{100 \mathrm{GeV}}\right)
$$

which neglected the co-annihilation channels.

Thus in AMSB the LSP does not give any problems with relic overabundance, but suffers the opposite problem - that sparticles in thermal equilibrium in the early universe will not produce sufficient neutralino dark matter. It has been suggested in [61] that decays of cosmological moduli - which are low mass particles predicted by string theory when supersymmetry is broken - could have produced Winos with sufficient abundance to be of astrophysical interest.

\subsection{Muon $g-2$}

It is well know that the gyromagnetic moment of the muon, $g_{\mu}$ can sensitive to sparticle interactions through loop corrections [62-66]. The recent BNL measurements $[67,68]$ dominate the world experimental average of the positive muon anomalous moment,

$$
a_{\mu}^{\mathrm{BNL}}=\left(g_{\mu}-2\right) / 2=11659203(8) \times 10^{-10} .
$$

Standard Model calculations of $a_{\mu}$ have been reviewed in $[69,70]$. Recent calculations $[71,72]$, which include a correction to the sign of the pion pole part of the hadronic light-by-light contribution [73-77], were combined in [68] to give:

$$
11659177(7) \times 10^{-10} \leq a_{\mu}^{\mathrm{SM}} \leq 11659186(8) \times 10^{-10} .
$$

The SUSY contribution is typically dominated by loops involving charginos and neutralinos $[69,78,79]$, and is proportional to $\tan \beta$ in the high $\tan \beta$ limit. The AMSB contributions to $a_{\mu}$ have been calculated in $[13,79]$. In fig. 15 we plot the $a_{\mu}^{\mathrm{AMSB}}$ for $\tan \beta=10$ and 30 , where again we have made use of the program micrOmegas. ${ }^{3}$ The AMSB contribution is small compared to both the experimental and theoretical uncertainties, except at small $m_{0}, m_{3 / 2}$.

\section{$6.3 B \rightarrow X_{s} \gamma$}

The inclusive radiative decay $B \rightarrow X_{s} \gamma$ is sensitive to sparticle properties through radiative corrections involving charged higgs, chargino and $\tilde{t}$ loops (see for example $[13,80-82])$.

\footnotetext{
${ }^{3}$ Note that ISASUSY produces AMSB points with negative $M_{1}$ and $M_{2}$ so the sign correlation between $\mu$ and $a_{\mu}^{\mathrm{SUSY}}$ is opposite to that in [79].
} 
Experimental measurements of the branching ratio from the CLEO [83], BELLE [84] and BaBar [85] collaborations give:

$$
\begin{aligned}
\mathcal{B}\left(B \rightarrow X_{s} \gamma\right)^{\mathrm{CLEO}} & =3.21 \pm 0.43 \pm 0.27_{-0.10}^{+0.18} \times 10^{-4} \\
\mathcal{B}\left(B \rightarrow X_{s} \gamma\right)^{\mathrm{BELLE}} & =3.39 \pm 0.53 \pm 0.42_{-0.55}^{+0.51} \times 10^{-4} \\
\mathcal{B}\left(B \rightarrow X_{s} \gamma\right)^{\mathrm{BaBar}} & =3.88 \pm 0.36 \pm 0.37_{-0.23}^{+0.43} \times 10^{-4}
\end{aligned}
$$

where the errors are statistical, systematic, and from theory respectively. Renormalisation scale uncertainties [86] lead to a $10 \%\left( \pm 0.3 \times 10^{-4}\right)$ uncertainty in the SM prediction. The mAMSB contributions were calculated using micrOmegas and are plotted in fig. 16. The combined experimental and theoretical uncertainty does not allow us to constrain the mAMSB parameter space at better than $2 \sigma$ for either of $\tan \beta=10,30$.

The overall effect of the $B \rightarrow X_{s} \gamma$ and to a greater extent the $g_{\mu}-2$ constraint is to disfavour the low $m_{0}, m_{3 / 2}$ region especially when $\tan \beta$ is large.

\section{Conclusions}

If anomaly mediated supersymmetry is present at the 1 to $2 \mathrm{TeV}$ scale, the LHC will observe excesses in various multi-lepton $+\mathbb{E}_{T}$ channels. We have used generic supersymmetry search procedures, and a realistic detector simulation, to investigate the ability of the experiments at the LHC to discover AMSB scenarios. By selecting events with very large missing energy and identifying tracks from chargino decays, the Wino-like nature of the LSP can be determined, and $m_{\tilde{\chi}_{1}^{+}}-m_{\tilde{\chi}_{1}^{0}}$ can be measured over a large range in parameter space. Careful study of the tracks left by $\tilde{\chi}_{1}^{ \pm} \mathrm{s}$ and their decay products can give clear evidence for this class of models even in cases where the chargino is shorter-lived than predicted in minimal AMSB.

\section{Acknowledgments}

The authors would like to thank Bryan Webber and Phil Stephens for providing constructive comments. We are indebted to Carolina Ödman and Phil Marshall of the Cavendish Astrophysics group who provided many of the references in sec. 6. We have made use of the ATLAS physics analysis framework and tools which are the result of collaboration-wide efforts. This work was partly funded by the UK Particle Physics and Astronomy Research Council. 


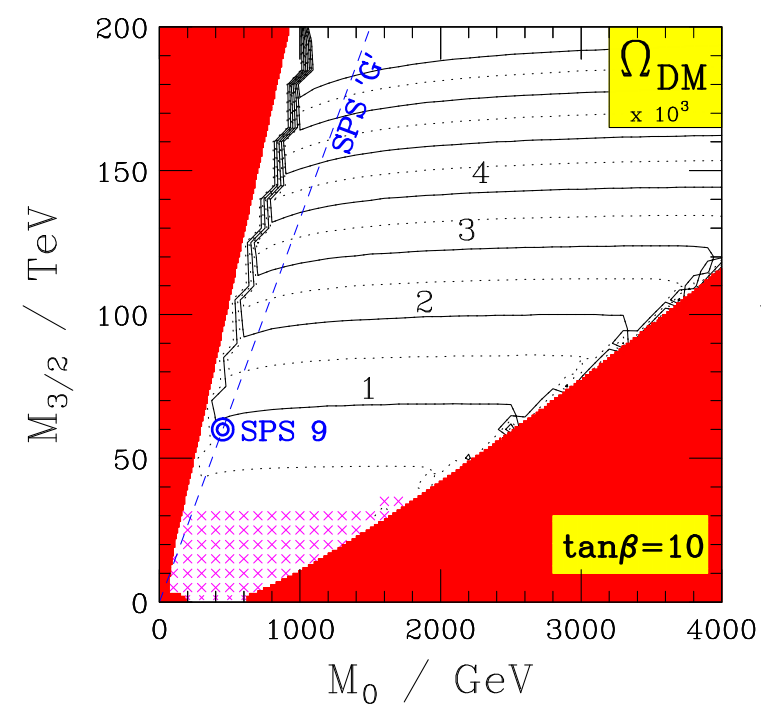

(a)

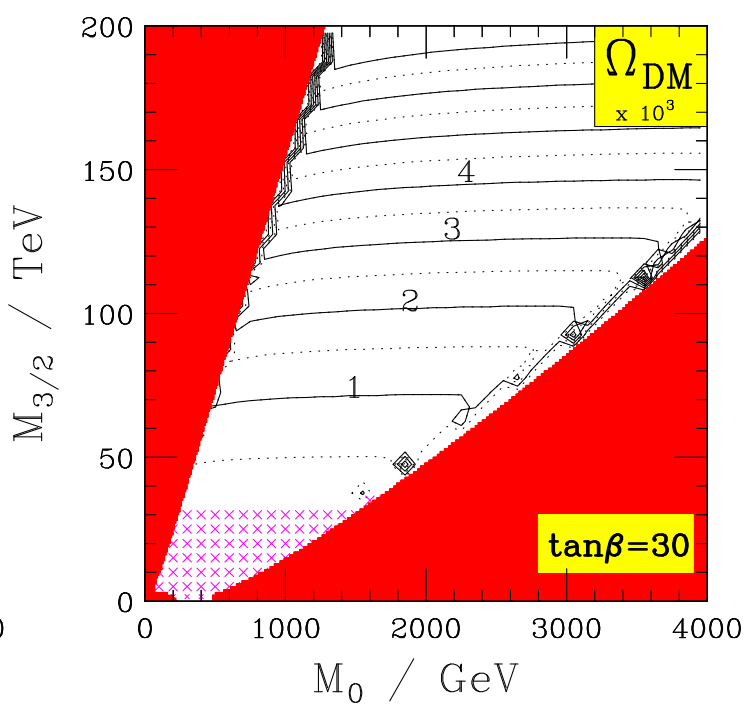

(b)

Figure 14: The relic density of the lightest neutralino, $\Omega_{\mathrm{CDM}} h^{2}$ with $\mu>0$; (a) for $\tan \beta=10$ and (b) for $\tan \beta=30$. The meaning of the symbols is explained in fig. 6. The astrophysical limits on $\Omega_{\mathrm{CDM}} h^{2}$ are discussed in the text. The contours are in units of $10^{-3}$.

\begin{tabular}{|l|r||l|c|}
\hline$\tilde{\chi}_{1}^{0} \tilde{\chi}_{1}^{0} \rightarrow W^{+} W^{-}$ & $8 \%$ & $\tilde{\chi}_{1}^{+} \tilde{\chi}_{1}^{0} \rightarrow$ fermion pair & $40 \%$ \\
$\tilde{\chi}_{1}^{+} \tilde{\chi}_{1}^{0} \rightarrow W^{+}+Z^{0} / h / A$ & $11 \%$ & $\tilde{\chi}_{1}^{+} \tilde{\chi}_{1}^{+} \rightarrow W^{+} W^{+}$ & $8 \%$ \\
$\tilde{\chi}_{1}^{+} \tilde{\chi}_{1}^{-} \rightarrow$ fermion pair & $15 \%$ & $\tilde{\chi}_{1}^{+} \tilde{\chi}_{1}^{-} \rightarrow$ boson pair & $12 \%$ \\
\hline
\end{tabular}

Table 6: The main cosmological relic annihilation channels for the Snowmass point SPS 9. 


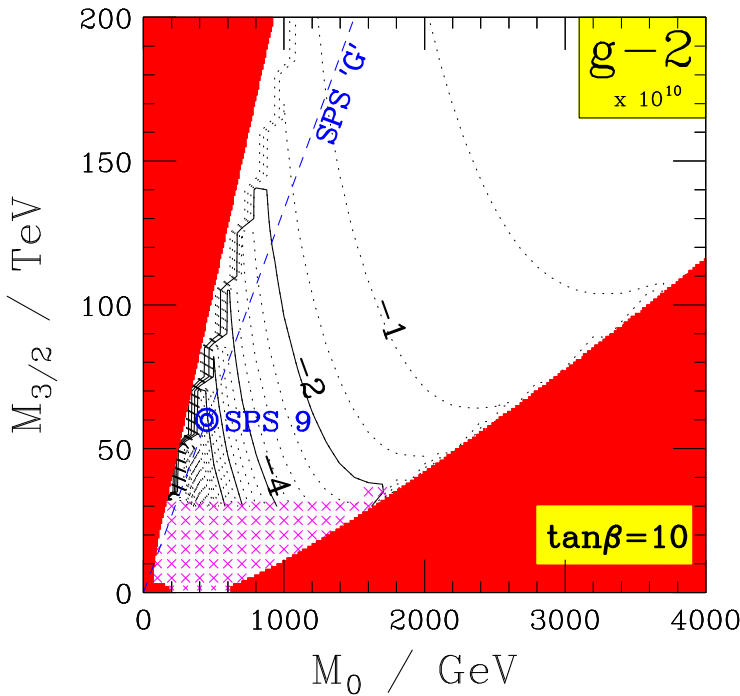

(a)

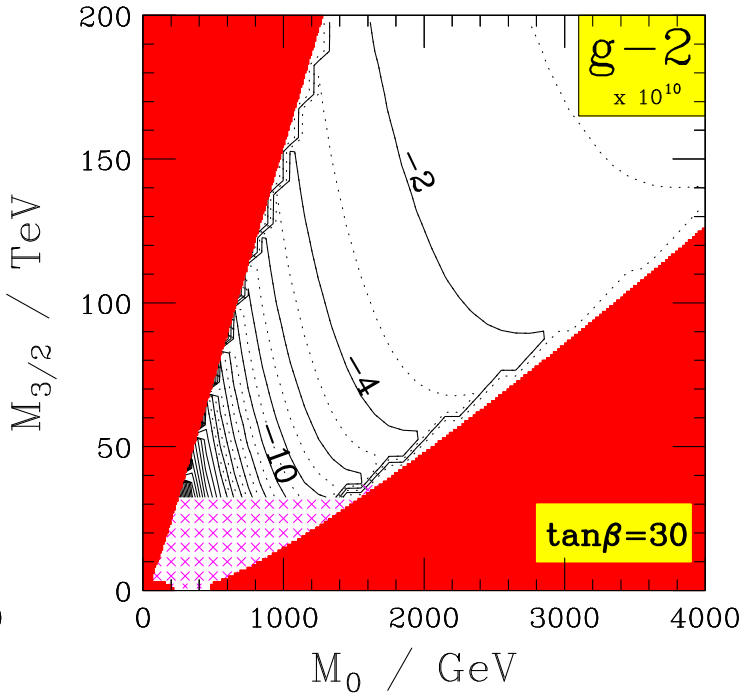

(b)

Figure 15: The SUSY contribution to the anomalous magnetic moment of the muon, (in units of $10^{-10}$ ) with $\mu>0$; (a) for $\tan \beta=10$ and (b) for $\tan \beta=30$. The meaning of the symbols is explained in fig. 6 .

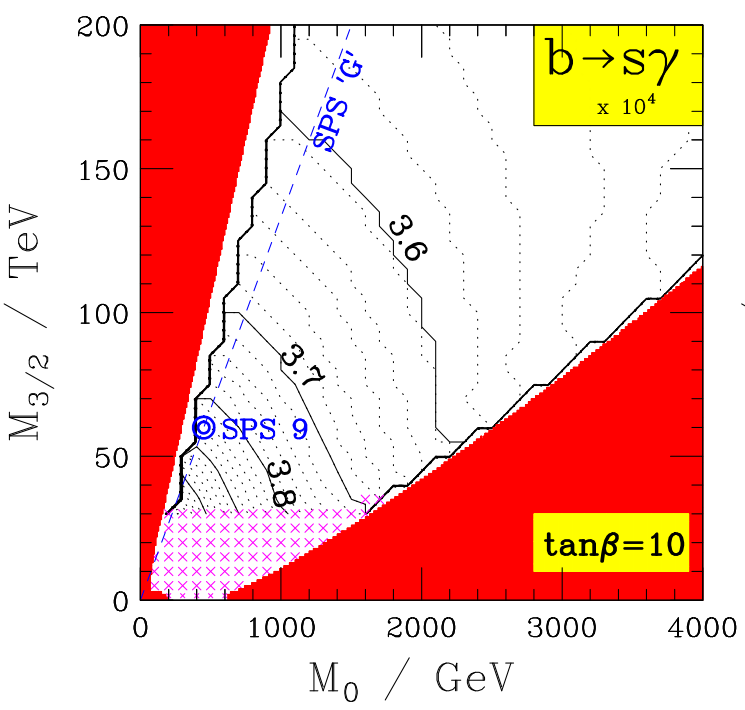

(a)

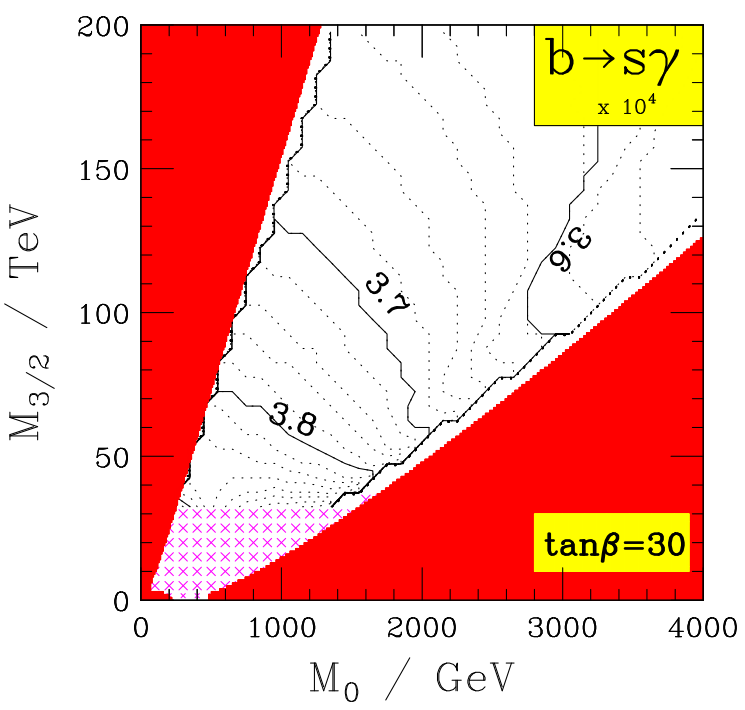

(b)

Figure 16: The branching ratio $\mathcal{B}\left(B \rightarrow X_{s} \gamma\right)$ with $\mu>0$; (a) for $\tan \beta=10$ and (b) for $\tan \beta=30$. The contours are in units of $10^{-4}$. The meaning of the symbols is explained in fig. 6 . 


\section{A. The variable $m_{T 2}$ and its generalisation}

The Cambridge $m_{T 2}$ variable, proposed in [87], can be used in analyses such as [23], where particles are pair-produced at hadronic colliders, and decay semi-invisibly, as is the case in R-parity conserving SUSY. This variable is used in symmetrical, twobody decays of supersymmetric particles, where the LSP is unobserved, and so must be inferred from missing energy.

\section{A.1 The properties of $m_{T 2}$}

For events in which the decay of a heavy object produces an unseen particle, such as

$$
\tilde{\chi}_{1}^{+} \rightarrow \tilde{\chi}_{1}^{0} \pi^{+}
$$

one can write the Lorentz invariant

$$
m_{\tilde{\chi}_{1}^{+}}^{2}=m_{\pi}^{2}+m_{\tilde{\chi}_{1}^{0}}^{2}+2\left[E_{T}^{\pi} E_{T}^{\tilde{\chi}_{1}^{0}} \cosh (\Delta \eta)-\mathbf{p}_{T}^{\pi} \cdot \mathbf{p}_{T}^{\tilde{\chi}_{1}^{0}}\right]
$$

where $\mathbf{p}_{T}^{\pi}$ and $\mathbf{p}_{T}^{\tilde{\chi}_{1}^{0}}$ indicate pion and neutralino 2-vectors in the transverse plane, and the transverse energies are defined by

$$
E_{T}^{\pi}=\sqrt{\left(\mathbf{p}_{T}^{\pi}\right)^{2}+m_{\pi}^{2}} \quad \text { and } \quad E_{T}^{\tilde{\chi}_{1}^{0}}=\sqrt{\left(\mathbf{p}_{T}^{\tilde{\chi}_{1}^{0}}\right)^{2}+m_{\tilde{\chi}_{1}^{0}}^{2}}
$$

Also

$$
\eta=\frac{1}{2} \log \left[\frac{E+p_{z}}{E-p_{z}}\right]
$$

is the true rapidity, so that

$$
\tanh \eta=p_{z} / E, \quad \sinh \eta=p_{z} / E_{T}, \quad \cosh \eta=E / E_{T} .
$$

In a hadron collider, only the transverse components of a missing particle's momentum can be inferred, so it is useful to define the transverse mass,

$$
m_{T}^{2}\left(\mathbf{p}_{T}^{\pi}, \mathbf{p}_{T}^{\tilde{\chi}_{1}^{0}}\right) \equiv m_{\pi^{+}}^{2}+m_{\tilde{\chi}_{1}^{0}}^{2}+2\left(E_{T}^{\pi} E_{T}^{\tilde{\chi}_{1}^{0}}-\mathbf{p}_{T}^{\pi} \cdot \mathbf{p}_{T}^{\tilde{\chi}_{1}^{0}}\right)
$$

which, because $\cosh (x) \geq 1$, is less than or equal to the mass of the lightest chargino, with equality only when the rapidity difference between the neutralino and the pion, $\Delta \eta_{\tilde{\chi}_{1}^{0} \pi}$ is zero. All other $\Delta \eta$ lead to $m_{T}<m_{\tilde{\chi}_{1}^{+}}$, so if we knew the neutralino momentum we could use $m_{T}$ to give an event by event lower bound on the lightest chargino mass. $m_{T}$ was used in this way by UA1 [88] in the measurement of the $W^{ \pm}$ mass. 
In R-parity conserving SUSY events there are expected to be two unseen LSPs. ${ }^{4}$ Since only the sum of the missing transverse momentum of the two neutralinos is known, the variable

$$
m_{T 2}^{2} \equiv \min _{\boldsymbol{q}_{T}^{(1)}+\mathbf{q}_{T}^{(2)}=\mathbf{p}_{T}}\left[\max \left\{m_{T}^{2}\left(\mathbf{p}_{T}^{\pi^{(1)}}, \boldsymbol{\phi}_{T}^{(1)}\right), m_{T}^{2}\left(\mathbf{p}_{T}^{\pi^{(2)}}, \boldsymbol{q}_{T}^{(2)}\right)\right\}\right]
$$

is a lower bound on the transverse mass $m_{T}$ for events where two decays of the type (A.1) occur. In (A.7) we have been forced to minimise over all consistent neutralino 2-momenta. Note that $\boldsymbol{\phi}_{T}^{(i)}$ is the hypothesised momentum of the $i$ th neutralino which need not be equal to its true momentum.

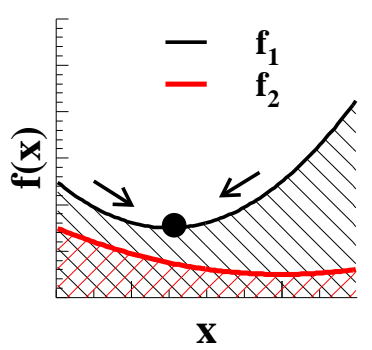

(a)

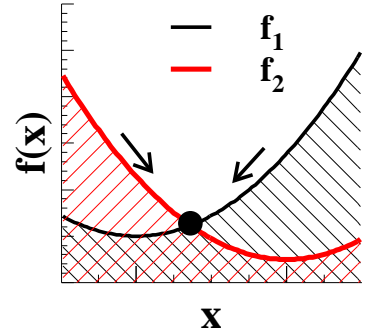

(b)

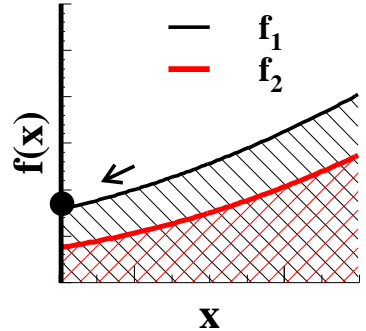

(c)

Figure 17: A diagram demonstrating that the minimisation over some parameter of the maximum of two well-behaved functions may occur either at (a) a minimum value of one of them, or (b) when they are equal, or (c) at the boundary of the domain.

To find the range of values $m_{T 2}$ may take we first let $f_{1}=m_{T}^{2}\left(\mathbf{p}_{T}^{\pi^{(1)}}, \boldsymbol{\phi}_{T}^{(1)}\right)$, and $f_{2}=m_{T}^{2}\left(\mathbf{p}_{T}^{\pi^{(2)}}, \boldsymbol{\phi}_{T}^{(2)}\right)$. We then note that the minimum over a parameter $x$ of the maximum of $f_{1}(x)$ and $f_{2}(x)$ can occur at a local minimum, $f_{1(2)}^{\prime}\left(x^{*}\right)=0$, provided $f_{1(2)}\left(x^{*}\right)>f_{2(1)}\left(x^{*}\right)$, as shown in fig. 17a. Alternatively the minimum can occur when the functions cross one another when $f_{1}=f_{2}$ (fig. 17b) or at a boundary (fig. 17c). The parameter $x$ corresponds to the fraction of the the missing momentum (in one of the transverse directions) which is assigned to each half of the event. Since $f_{1}, f_{2} \rightarrow \infty$ as $x \rightarrow \pm \infty$ fig. $17 \mathrm{c}$ is not relevant to our minimisation problem.

To see that case (a) cannot occur, consider the unconstrained minimisation over $\boldsymbol{\phi}_{T}$, of $m_{T}^{2}\left(\mathbf{p}_{T}^{\pi}, \boldsymbol{\phi}_{T}\right)$. Using the relationship

$$
\frac{\partial \mathbb{E}_{T}}{\partial \phi_{k}}=\frac{\not q_{k}}{\mathbb{E}_{T}}
$$

where $\mathbb{E}_{T}^{2}=\boldsymbol{\phi}_{T}^{2}+m_{\tilde{\chi}_{1}^{0}}^{2}$, it is straightforward to show that,

$$
\frac{\partial m_{T}^{2}}{\partial \phi_{k}}=2\left(E_{T}^{\pi} \frac{\not q_{k}}{\not_{T}}-p_{k}^{\pi}\right) \quad k=1,2 .
$$

\footnotetext{
${ }^{4}$ Though there may also be other unseen particles - see appendix A.2.
} 
This means that at the minimum

$$
\mathbf{v}_{T}^{\pi}=\boldsymbol{\mu}_{T}
$$

where we introduce the notation $\mathbf{v}_{T} \equiv \mathbf{p}_{T} / E_{T}, \boldsymbol{\mu}_{T} \equiv \boldsymbol{\phi}_{T} / \mathbb{E}_{T}$. where $\mathbf{p}_{T}$ and $\mathbf{v}_{T}$ represent the true transverse momentum and velocity of a particle, while $\boldsymbol{\phi}_{T}$ and $\boldsymbol{\mu}_{T}$ are assigned by the minimisation.

Using the basis $(t, x, y)$ with the metric $\operatorname{diag}(1,-1,-1)$, one can write

$$
m_{T}^{2}=\left(E_{T}^{\mathrm{tot}}, \mathbf{p}_{T}^{\mathrm{tot}}\right) \cdot\left(E_{T}^{\mathrm{tot}}, \mathbf{p}_{T}^{\mathrm{tot}}\right),
$$

where $E_{T}^{\text {tot }}=E_{T}^{\pi}+\mathbb{E}_{T}$ and $\mathbf{p}_{T}^{\text {tot }}=\mathbf{p}_{T}^{\pi}+\boldsymbol{\phi}_{T}$. This $1+2$ dimensional Lorentz invariant can be evaluated in any frame boosted from the lab in the transverse plane. (A.10) has told us that at the unconstrained minimum the transverse velocities $\mathbf{v}_{T}^{\pi}$ and $\boldsymbol{\psi}_{T}$ are equal; a statement necessarily true in all transverse frames, including the special one in which both the transverse velocities (and associated momenta) are zero. Evaluating (A.11) in this frame, we find that the unconstrained minimum of (A.11) then becomes $\left(m_{\pi}+m_{\tilde{\chi}_{1}^{0}}, 0,0\right) \cdot\left(m_{\pi}+m_{\tilde{\chi}_{1}^{0}}, 0,0\right)$, and we recover the expected result

$$
m_{T}^{\min }=m_{\pi}+m_{\tilde{\chi}_{1}^{0}}
$$

We therefore conclude that the function $m_{T}^{2}$ has only one stationary value and it is the global minimum, and is common to both sides of the event provided the same type of particles are emitted. Thus when $f_{1}$ is minimum it cannot be greater than $f_{2}$, and so the minimisation in (A.7) forces $f_{1}=f_{2}$. This could of course occur when both $f_{1}$ and $f_{2}$ are at their global minima, in which case $m_{T 2}$ takes its minimum value:

$$
m_{T 2}{ }^{\min }=m_{\pi}+m_{\tilde{\chi}_{1}^{0}}
$$

To summarise, $m_{T 2}$ is the minimum of $m_{T}^{(1)}$ subject to the two constraints $m_{T}^{(1)}=$ $m_{T}^{(2)}$, and $\boldsymbol{p}_{T}^{(1)}+\boldsymbol{p}_{T}^{(2)}=\boldsymbol{p}_{T}$. The condition for the minimisation can be calculated by Lagrange multiplier methods, the result of which is that the velocity vectors $\boldsymbol{\mu}_{T}^{(1,2)}$ of the assigned neutralino momenta $\boldsymbol{\phi}_{T}^{(1,2)}$ must satisfy

$$
\left(\mathbf{\mu}_{T}^{(1)}-\mathbf{v}_{T}^{\pi^{(1)}}\right) \propto\left(\dot{\mu}_{T}^{(2)}-\mathbf{v}_{T}^{\pi^{(2)}}\right) .
$$

To find the maximum of $m_{T 2}$ over many events we note that for each event the minimisation will select hypothesised momenta satisfying (A.14). We now note events can occur in which the true transverse velocities of the neutralinos were exactly those which were assigned by the minimisation, i.e. they can satisfy

$$
\mathbf{v}_{T}^{\tilde{\chi}_{1}^{0}(1)}=\boldsymbol{\mu}_{T}^{(1)}, \quad \mathbf{v}_{T}^{\tilde{1}_{1}^{0}(2)}=\boldsymbol{\mu}_{T}^{(2)}
$$


These events will have both hypothesised transverse masses equal not only to each other but also to true transverse masses which would have been calculated if the neutralino momenta had been known:

$$
m_{T}^{(i)}\left(\mathbf{p}_{T}^{\pi^{(i)}}, \boldsymbol{p}_{T}^{\tilde{\chi}_{1}^{0}(i)}\right)=m_{T}^{(i)}\left(\mathbf{p}_{T}^{\pi^{(i)}}, \boldsymbol{\phi}_{T}^{(i)}\right)
$$

If events occur where, in addition to the transverse components of the neutralino momenta satisfying (A.15), the rapidity differences satisfy $\eta_{\tilde{\chi}_{1}^{0}(1)}=\eta_{\pi(1)}$ and $\eta_{\tilde{\chi}_{1}^{0}(2)}=$ $\eta_{\pi(2)}$, then by (A.2) $m_{T 2}$ will equal the true mass of the chargino. Combining this with (A.13) and recalling that $m_{T 2}$ cannot be greater than the chargino mass by construction, we can see that the event-by event distribution of $m_{T 2}$ can span the range

$$
m_{\tilde{\chi}_{1}^{0}}+m_{\pi} \leq m_{T 2} \leq m_{\tilde{\chi}_{1}^{+}}
$$

showing that $m_{T 2}$ is sensitive to the $m_{\tilde{\chi}_{1}^{+}}-m_{\tilde{\chi}_{1}^{0}} \equiv \Delta M_{\tilde{\chi}_{1}}$ mass difference.

The variable is equally applicable to two same-sign $\tilde{\chi}_{1}^{+}$decays so $m_{T 2}$ signal events can be defined as those having two $\tilde{\chi}_{1}^{ \pm} \rightarrow \tilde{\chi}_{1}^{0} \pi^{ \pm}$decays with any combination of charges.

\section{A.2 Generalisations of $m_{T 2}$}

In our analysis we also wish to make use of the leptonic decays $\tilde{\chi}_{1}^{+} \rightarrow \tilde{\chi}_{1}^{0} \ell \nu_{\ell}$ where $\ell \in e, \mu$. We therefore generalise $m_{T 2}$ to cases where more than two particles go undetected.

Consider events in which a chargino is produced and then decays to $\tilde{\chi}_{1}^{0}$ e $\nu_{e}$. If we expand the Lorentz invariant

$$
\left(m_{\tilde{\chi}_{1}^{+}}\right)^{2}=\left(p_{\tilde{\chi}_{1}^{0}}+p_{e}+p_{\nu}\right)^{2}
$$

we obtain three mass-squared terms for each of the decay particles and three crossterms. The cross-terms can each be written in the form

$$
p_{a} \cdot p_{b}=E_{T}^{(a)} E_{T}^{(b)} \cosh \left(\Delta \eta_{a b}\right)-\mathbf{p}_{T}^{(a)} \cdot \mathbf{p}_{T}^{(b)}
$$

If the neutralino and neutrino transverse momenta were individually known we could evaluate the transverse mass,

$m_{T}^{2}=m_{\tilde{\chi}_{1}^{0}}^{2}+m_{e}^{2}+2\left[\left(E_{T}^{e} E_{T}^{\chi}-\mathbf{p}_{T}^{e} \cdot \mathbf{p}_{T}^{\chi}\right)+\left(E_{T}^{\nu} E_{T}^{\chi}-\mathbf{p}_{T}^{\nu} \cdot \mathbf{p}_{T}^{\chi}\right)+\left(E_{T}^{e} E_{T}^{\nu}-\mathbf{p}_{T}^{e} \cdot \mathbf{p}_{T}^{\nu}\right)\right]$,

where the neutrino mass is assumed to be negligible. $m_{T}$ will be equal to the $\tilde{\chi}_{1}^{+}$ mass in events where $\Delta \eta_{a b}=0$ for all pairs of $e, \nu_{e}$, and $\tilde{\chi}_{1}^{0}$. 


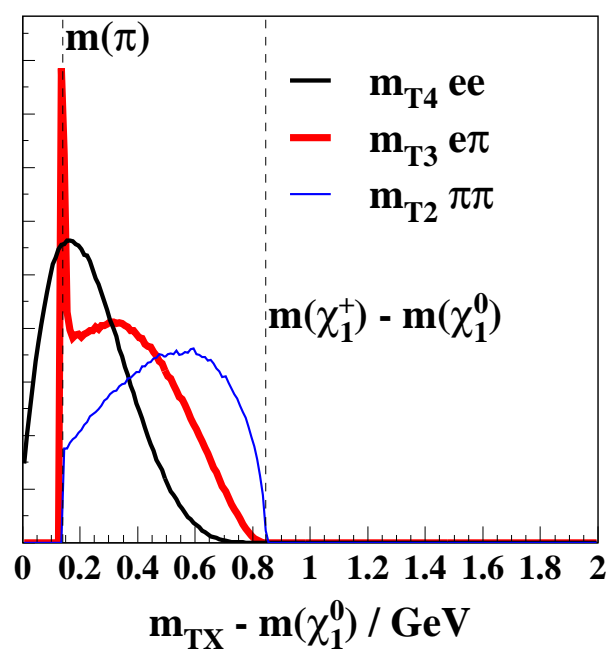

(a)

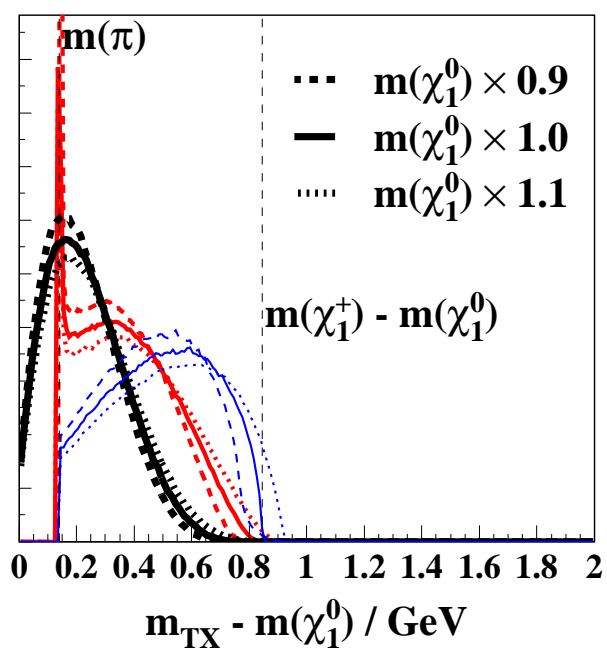

(b)

Figure 18: (a) Simulations of $m_{T X}-m_{\tilde{\chi}_{1}^{0}}$ for $X=2,3,4$ using a simple phase-space Monte-Carlo generator program for a pair of decays $\tilde{q} \rightarrow \tilde{\chi}_{1}^{+} q$ followed by $\tilde{\chi}_{1}^{+} \rightarrow \tilde{\chi}_{1}^{0} \pi$ or $\tilde{\chi}_{1}^{+} \rightarrow \tilde{\chi}_{1}^{0}$ e $\nu_{e}$. As the number of invisible particles increases the proportion of events near the upper limit decreases. The peak in $m_{T 3}-m_{\tilde{\chi}_{1}^{0}}$ near the pion mass is explained in the text. (b) The distortion of $m_{T X}-m_{\tilde{\chi}_{1}^{0}}$ when the LSP mass is varied by $\pm 10 \%$, showing that $M_{T X}-m_{\tilde{\chi}_{1}^{0}}$ remains sensitive to the mass difference $\Delta M_{\tilde{\chi}_{1}}=m_{\tilde{\chi}_{1}^{+}}-m_{\tilde{\chi}_{1}^{0}}$. In this simulation $\Delta M_{\tilde{\chi}_{1}}=0.845 \mathrm{GeV}, m_{\tilde{\chi}_{1}^{0}}=161.6 \mathrm{GeV}$, and the electron and neutrino mass were neglected. The normalisation is arbitrary.

In events with two leptonic chargino decays a variable like $m_{T 2}$ can be defined as in (A.7) but using the three-particle definition of $m_{T}$ from (A.20) and with the modified constraint,

$$
\mathbf{q}_{T}^{\nu(1)}+\mathbf{q}_{T}^{\chi(1)}+\mathbf{q}_{T}^{\nu(2)}+\mathbf{q}_{T}^{\chi(2)}=\boldsymbol{p}_{T}
$$

where the labels (1) and (2) indicate which chargino the particles were emitted from. We call this variable $m_{T 4}$ (or indeed $m_{T X}$ where $X$ is the number of undetected particles).

The conditions for the minimisation required to calculate $m_{T 4}$ can be calculated just as for $m_{T 2}$. The Euler-Lagrange equations involving

$$
\frac{\partial\left(m_{T}^{(i)}\right)^{2}}{\partial \mathbf{q}_{T}^{\nu(i)}} \quad \text { and } \quad \frac{\partial\left(m_{T}^{(i)}\right)^{2}}{\partial \mathbf{q}_{T}^{\tilde{\chi}_{1}^{0}(i)}}
$$

show that the minimisation will select the invisible particles' momenta such that $\mathbf{u}_{T}^{\tilde{\chi}_{1}^{0}(i)}=\mathbf{u}_{T}^{\nu(i)}$. The other E-L equations reproduce (A.14) but with electrons replacing pions.

This means that when calculating $m_{T 4}$ one can replace the missing particles from each chargino decay with a pseudo-particle with mass equal to the sum of the masses 
of those invisible particles and proceed as for $m_{T 2}$. In the case of leptonic chargino decay the mass of the neutrino can be safely neglected in comparison to that of the $\tilde{\chi}_{1}^{0}$, and the constraint $\mathbf{u}_{T}^{\chi(i)}=\mathbf{u}_{T}^{\nu(i)}$ is equivalent to $\mathbf{q}_{T}^{\nu(i)}=(0,0)$.

The generalisation to $m_{T X}$ for other values of $X$ is straightforward. The distribution of $m_{T 3}$ is shown in fig. 18a for events in which one chargino decays to $\tilde{\chi}_{1}^{0}, e, \nu$ and another to $\tilde{\chi}_{1}^{0}, \pi^{+}$. Unlike $m_{T 2}$ and $m_{T 4}$ it has a sharp peak at $m_{T 3}=m_{\tilde{\chi}_{1}^{0}}+m_{\pi}$. This occurs because the visible particles on each side of the event are different and so the unconstrained minimum of the values of $m_{T}$ on each side of the event are not equal as they are in the case of $m_{T 2}$ and $m_{T 4}$ :

$$
\min _{\boldsymbol{\phi}_{\mathbf{T}}^{(\mathbf{( 1 )}}}\left(m_{T}^{(1)}\left(\mathbf{p}_{T}^{\pi}, \boldsymbol{\phi}_{T}^{(1)}\right)\right)=m_{\pi}+m_{\tilde{\chi}_{1}^{0}} \neq m_{e}+m_{\tilde{\chi}_{1}^{0}}=\min _{\boldsymbol{\phi}_{\mathbf{T}}^{(\mathbf{2})}}\left(m_{T}^{(2)}\left(\mathbf{p}_{T}^{e}, \boldsymbol{\phi}_{T}^{(2)}\right)\right)
$$

Some of the events can then fall into the category shown in fig. 17a, producing a peak of events with $m_{T}=m_{\tilde{\chi}_{1}^{0}}+m_{\pi}$.

The distribution over events of $m_{T 4}$ will have fewer entries near the upper kinematic limit $\left(m_{T 4}=m_{\tilde{\chi}_{1}^{+}}\right)$because when more particles go undetected an event at that limit must satisfy a larger number of constraints. For fully leptonic chargino decay, there are six constraints of the type $\Delta \eta=0$, two $\mathbf{p}_{T}^{\nu(i)}=0$ and finally the modified constraint from (A.14). This effect can be seen in fig. 18a for events where a total of two, three and four invisible particles are produced.

A further generalisation which we do not require here might be relevant in cases where more than one visible particle is emitted from each mother. For such decays, one would sum the full 4-momenta of the visible particles from each decay as well as summing the masses of the invisible particles from each side of the event and proceed as for $m_{T 2}$.

\section{A.3 Uncertainties in $\not p_{T}$ and $m_{\tilde{\chi}_{1}^{0}}$}

The sensitivity of $m_{T X}$ to the estimated mass of the neutralino is shown in fig. $18 \mathrm{~b}$, where $10 \%(16 \mathrm{GeV})$ errors in $\chi$ result in similar fractional errors in $\Delta M_{\tilde{\chi}_{1}}$ i.e. of a few tens of $\mathrm{MeV} . m_{T X}$ shows similar insensitivity to measurement uncertainties in the missing transverse momentum vector. This behaviour can be (at least partially) understood from the non-relativistic limit of $m_{T 2}$, when the proportionality in (A.14) becomes an equality and

$$
m_{T 2}{ }^{2}-\left(m_{\pi}+m_{\tilde{\chi}_{1}^{0}}\right)^{2}=\frac{1}{4 m_{\pi} m_{\tilde{\chi}_{1}^{0}}}\left(m_{\pi} \boldsymbol{p}_{T}-m_{\tilde{\chi}_{1}^{0}} \mathbf{p}_{T}^{\pi_{1}}-m_{\tilde{\chi}_{1}^{0}} \mathbf{p}_{T}^{\pi_{2}}\right)^{2}+\mathcal{O}\left(\left(\mathbf{v}_{T} \cdot \mathbf{v}_{T}\right)^{2}\right)
$$

One can see that in (A.23) $m_{\pi}$ multiplies the missing momentum, while $m_{\tilde{\chi}_{1}^{0}}$ multiplies the pion transverse momenta. 


\section{B. Simulation of particle tracks}

Part of the ATLFAST [38] software provides fast parameterised simulation of the ATLAS inner detector performance. In particular it calculates the expected track reconstruction efficiency, and smears the five track helix parameters and according to particle type. The parameterisation used was based on GEANT3 Monte-Carlo simulations [39] using a Kalman-filter algorithm for track reconstruction [89]. A parameterisation from a large statistics Monte-Carlo sample [90], was used for the hadronic track smearing for this study.

\section{B.1 Low $p_{T}$ electron identification}

In ATLAS the transition radiation tracker allows the identification of low-energy electrons because they emit more transition radiation than more massive particles with the same momentum. The issue of particle misidentification is not dealt with in the standard version of ATLFAST, and so has been implemented independently in our analysis.

\begin{tabular}{|c|c|c|c|c|c|c|}
\hline$p_{T}$ & 0.5 & 1 & 2 & 5 & 10 & $\geq 20$ \\
\hline$\epsilon_{\pi}$ & 0.01 & 0.003 & 0.005 & 0.007 & 0.008 & 0.012 \\
\hline
\end{tabular}

Table 7: Pion misidentification efficiency at $|\eta|=0.3$ as a function of transverse momentum for an electron identification efficiency of $90 \%$. Interpolations were made linearly in $p_{T}$ and logarithmically in $\epsilon_{\pi}$. Particles with $p_{T}<0.5 \mathrm{GeV}$ are assumed not to be reconstructed into tracks.

\begin{tabular}{|cc|c|c|c|c|c|c|c|c|c|}
\hline & $|\eta|$ & $\leq 0.2$ & 0.3 & 0.65 & 0.8 & 1.15 & 1.4 & 1.9 & 2.15 & $\geq 2.4$ \\
\hline$p_{T}$ & $2 \mathrm{GeV}$ & 1.2 & 1.0 & 0.88 & 1. & 0.6 & 0.4 & 0.028 & 0.88 & 2 \\
\hline$p_{T}$ & $20 \mathrm{GeV}$ & 1.1 & 1.0 & 1. & 2.0 & 1.4 & 0.78 & 0.22 & 1.4 & 2.3 \\
\hline
\end{tabular}

Table 8: $\eta$ dependent correction factor applied to $\epsilon_{\pi}$, the hadron misidentification efficiency, for two different values of transverse momentum. Interpolations were made logarithmically in $\epsilon_{\pi}$ and linearly in $p_{T}$ and $\eta$. Tracks beyond $|\eta|=2.5$ are not reconstructed.

Test beam performance was compared to Monte-Carlo simulations in [91] using a combined discriminator which considered both the number of high threshold clusters and the time for which the charge deposited exceeded a lower threshold. In that paper the pion misidentification probability was calculated as a function of transverse momentum at $\eta=0.3$ for an electron efficiency of $90 \%$ (table 7). For our simulation these efficiencies were extrapolated to other values of $\eta$ by comparison with the 
GEANT3 simulations performed in [22]. The $p_{T}$ and $\eta$ dependent correction factors are shown in table 8 .

\section{B.2 Low $p_{T}$ muon identification}

High energy muons are easily identified because of their high penetration. Identification efficiency is drastically reduced when muons have an insufficient transverse momentum to extend their track helix into the dedicated muon detectors. The applied identification efficiencies for muons were based on [22] and are shown in table 9 .

\begin{tabular}{|l|c|c|c|c|c|c|c|}
\hline$p_{T}$ & $\leq 2$ & 3 & 4 & 5 & 6 & 8 & $\geq 10$ \\
\hline Efficiency & 0 & 0.34 & 0.66 & 0.89 & 0.95 & 0.96 & 0.98 \\
\hline
\end{tabular}

Table 9: Applied muon identification efficiency as a function of transverse momentum. Efficiencies were interpolated linearly in $\epsilon_{\mu}$ and $p_{T}$. 


\section{References}

[1] S. Dimopoulos and G. F. Giudice, Naturalness constraints in supersymmetric theories with nonuniversal soft terms, Phys. Lett. B357 (1995) 573-578, http://arXiv.org/abs/hep-ph/9507282.

[2] G. W. Anderson and D. J. Castano, Naturalness and superpartner masses or when to give up on weak scale supersymmetry, Phys. Rev. D52 (1995) 1693-1700, http://arXiv.org/abs/hep-ph/9412322.

[3] L. Randall and R. Sundrum, Out of this world supersymmetry breaking, Nucl. Phys. B557 (1999) 79-118, http://arXiv.org/abs/hep-th/9810155.

[4] G. F. Giudice, M. A. Luty, H. Murayama, and R. Rattazzi, Gaugino mass without singlets, JHEP 12 (1998) 027, http://arXiv.org/abs/hep-ph/9810442].

[5] A. Pomarol and R. Rattazzi, Sparticle masses from the superconformal anomaly, JHEP 05 (1999) 013, [http://arXiv.org/abs/hep-ph/9903448].

[6] Z. Chacko, M. A. Luty, I. Maksymyk, and E. Ponton, Realistic anomaly-mediated supersymmetry breaking, JHEP 04 (2000) 001, http://arXiv.org/abs/hep-ph/9905390.

[7] E. Katz, Y. Shadmi, and Y. Shirman, Heavy thresholds, slepton masses and the mu term in anomaly mediated supersymmetry breaking, JHEP 08 (1999) 015, http://arXiv.org/abs/hep-ph/9906296.

[8] M. Carena, K. Huitu, and T. Kobayashi, RG-invariant sum rule in a generalization of anomaly mediated SUSY breaking models, Nucl. Phys. B592 (2001) 164-182, http://arXiv.org/abs/hep-ph/0003187.

[9] I. Jack and D. R. T. Jones, Fayet-Iliopoulos D-terms and anomaly mediated supersymmetry breaking, Phys. Lett. B482 (2000) 167-173, http://arXiv.org/abs/hep-ph/0003081.

[10] N. Arkani-Hamed, D. E. Kaplan, H. Murayama, and Y. Nomura, Viable ultraviolet-insensitive supersymmetry breaking, JHEP 02 (2001) 041, http://arXiv.org/abs/hep-ph/0012103.

[11] Z. Chacko, M. A. Luty, E. Ponton, Y. Shadmi, and Y. Shirman, The GUT scale and superpartner masses from anomaly mediated supersymmetry breaking, Phys. Rev. D64 (2001) 055009, http://arXiv.org/abs/hep-ph/0006047.

[12] T. Gherghetta, G. F. Giudice, and J. D. Wells, Phenomenological consequences of supersymmetry with anomaly-induced masses, Nucl. Phys. B559 (1999) 27-47, http://arXiv.org/abs/hep-ph/9904378. 
[13] J. L. Feng and T. Moroi, Supernatural supersymmetry: Phenomenological implications of anomaly-mediated supersymmetry breaking, Phys. Rev. D61 (2000) 095004, [http://arXiv.org/abs/hep-ph/9907319].

[14] K. Huitu, J. Laamanen, and P. N. Pandita, Sparticle spectrum and constraints in anomaly mediated supersymmetry breaking models, Phys. Rev. D65 (2002) 115003, http://arXiv.org/abs/hep-ph/0203186.

[15] J. L. Feng, T. Moroi, L. Randall, M. Strassler, and S.-f. Su, Discovering supersymmetry at the Tevatron in Wino LSP scenarios, Phys. Rev. Lett. 83 (1999) 1731-1734, http://arXiv.org/abs/hep-ph/9904250.

[16] B. C. Allanach and A. Dedes, R-parity violating anomaly mediated supersymmetry breaking, JHEP 06 (2000) 017, http://arXiv.org/abs/hep-ph/0003222.

[17] F. De Campos, M. A. Diaz, O. J. P. Eboli, M. B. Magro, and P. G. Mercadante, Anomaly mediated supersymmetry breaking without R-parity, Nucl. Phys. B623 (2002) 47-72, http://arXiv.org/abs/hep-ph/0110049.

[18] B. C. Allanach et. al., The Snowmass points and slopes: Benchmarks for SUSY searches, http://arXiv.org/abs/hep-ph/0202233.

[19] G. Corcella et. al., HERWIG 6: An event generator for hadron emission reactions with interfering gluons (including supersymmetric processes), JHEP 01 (2001) 010, hep-ph/0011363.

[20] S. Moretti, K. Odagiri, P. Richardson, M. H. Seymour, and B. R. Webber, Implementation of supersymmetric processes in the HERWIG event generator, JHEP 04 (2002) 028, http://arXiv.org/abs/hep-ph/0204123.

[21] G. Corcella et. al., Herwig 6.3 release note, http://arXiv.org/abs/hep-ph/0107071.

[22] ATLAS Detector and Physics Peformance TDR. No. CERN/LHCC/99-14, CERN/LHCC/99-15. CERN, 1999.

[23] B. C. Allanach, C. G. Lester, M. A. Parker, and B. R. Webber, Measuring sparticle masses in non-universal string inspired models at the LHC, JHEP 09 (2000) 004, hep-ph/0007009.

[24] F. E. Paige and J. Wells, Anomaly mediated SUSY breaking at the LHC, http://arXiv.org/abs/hep-ph/0001249.

[25] A. Djouadi et. al., Les Points d'Aix, 2001. Euro GDR SUSY workshop http://www.desy.de/ ${ }^{\sim}$ heinemey/LesPointsdAix.htm].

[26] ALEPH Collaboration, A. Heister et. al., Search for charginos nearly mass degenerate with the lightest neutralino in $e^{+} e^{-}$collisions at centre-of-mass energies up to 209-GeV, Phys. Lett. B533 (2002) 223-236, http://arXiv.org/abs/hep-ex/0203020. 
[27] D. K. Ghosh, A. Kundu, P. Roy, and S. Roy, Characteristic Wino signals in a linear collider from anomaly mediated supersymmetry breaking, Phys. Rev. D64 (2001) 115001, [http://arXiv.org/abs/hep-ph/0104217.

[28] D. K. Ghosh, P. Roy, and S. Roy, Linear collider signal of a Wino LSP in anomaly-mediated scenarios, JHEP 08 (2000) 031, http://arXiv.org/abs/hep-ph/0004127.

[29] H. Baer, J. K. Mizukoshi, and X. Tata, Reach of the CERN LHC for the minimal anomaly-mediated SUSY breaking model, Phys. Lett. B488 (2000) 367-372, http://arXiv.org/abs/hep-ph/0007073.

[30] C. H. Chen, M. Drees, and J. F. Gunion, A non-standard string/SUSY scenario and its phenomenological implications, Phys. Rev. D55 (1997) 330-347, http://arXiv.org/abs/hep-ph/9607421.

[31] C. H. Chen, M. Drees, and J. F. Gunion, Addendum/erratum for 'Searching for invisible and almost invisible particles at $e^{+} e^{-}$colliders' and 'A non-standard string/SUSY scenario and its phenomenological implications', http://arXiv.org/abs/hep-ph/9902309.

[32] J. H. Kuhn and A. Santamaria, Tau decays to pions, Z. Phys. C48 (1990) 445-452.

[33] F. E. Paige, S. D. Protopopescu, H. Baer, and X. Tata, ISAJET 7.40: A Monte Carlo event generator for $p p, \bar{p} p$, and $e^{+} e^{-}$reactions, hep-ph/9810440.

[34] H. Baer, F. E. Paige, S. D. Protopopescu, and X. Tata, Simulating supersymmetry with ISAJET 7.0 / ISASUSY 1.0, http://arXiv.org/abs/hep-ph/9305342.

[35] G. Corcella et. al., Herwig 6.4 release note, http://arXiv.org/abs/hep-ph/0201201.

[36] P. Richardson, Spin correlations in Monte Carlo simulations, JHEP 11 (2001) 029, http://arXiv.org/abs/hep-ph/0110108.

[37] W. T. Giele, T. Matsuura, M. H. Seymour, and B. R. Webber, W boson plus multijets at hadron colliders: Herwig parton showers versus exact matrix elements, . Contribution to Proc. of 1990 Summer Study on High Energy Physics: Research Directions for the Decade, Snowmass, CO, Jun 25 - Jul 13, 1990.

[38] E. Richter-Was, D. Froidevaux, and L. Poggioli, Atlfast 2.0: A fast simulation package for ATLAS, ATL-PHYS-98-131. ATLAS internal note.

[39] E. Buis et. al., Update of inner detector performance parameterisations, ATL-INDET-98-215. ATLAS internal note.

[40] CMS Collaboration, S. Abdullin et. al., Discovery potential for supersymmetry in CMS, J. Phys. G28 (2002) 469, http://arXiv.org/abs/hep-ph/9806366. 
[41] S. I. Bityukov and N. V. Krasnikov, The LHC (CMS) discovery potential for models with effective supersymmetry and nonuniversal gaugino masses, http://arXiv.org/abs/hep-ph/0102179.

[42] D. R. Tovey, Inclusive SUSY searches and measurements at ATLAS, 2002. ATLAS Scientific Note: SN-ATLAS-2002-020 [http://weblib.cern.ch/abstract?SN-ATLAS-2002-020].

[43] J. F. Gunion and S. Mrenna, A study of SUSY signatures at the Tevatron in models with near mass degeneracy of the lightest chargino and neutralino, Phys. Rev. D62 (2000) 015002, http://arXiv.org/abs/hep-ph/9906270.

[44] J. F. Gunion and S. Mrenna, Probing models with near degeneracy of the chargino and LSP at a linear $e^{+} e^{-}$collider, Phys. Rev. D64 (2001) 075002, http://arXiv.org/abs/hep-ph/0103167.

[45] S. Ambrosanio, B. Mele, S. Petrarca, G. Polesello, and A. Rimoldi, Measuring the SUSY breaking scale at the LHC in the slepton LSP scenario of GMSB models, JHEP 01 (2001) 014, [http://arXiv.org/abs/hep-ph/0010081].

[46] B. C. Allanach, C. M. Harris, M. A. Parker, P. Richardson, and B. R. Webber, Detecting exotic heavy leptons at the large hadron collider, JHEP 08 (2001) 051, http://arXiv.org/abs/hep-ph/0108097.

[47] ATLAS Pixel Detector TDR. No. CERN/LHCC 98-13. CERN, 1998.

[48] K. Einsweiler. Private communication.

[49] A. Brignole, L. E. Ibanez, and C. Munoz, Soft supersymmetry-breaking terms from supergravity and superstring models, http://arXiv.org/abs/hep-ph/9707209.

[50] H. E. Haber and G. L. Kane, The search for supersymmetry: Probing physics beyond the Standard Model, Phys. Rept. 117 (1985) 75.

[51] H.-C. Cheng, B. A. Dobrescu, and K. T. Matchev, Generic and chiral extensions of the supersymmetric Standard Model, Nucl. Phys. B543 (1999) 47-72, http://arXiv.org/abs/hep-ph/9811316.

[52] UA5 Collaboration, G. J. Alner et. al., The UA5 high-energy $\bar{p} p$ simulation program, Nucl. Phys. B291 (1987) 445.

[53] CDF Collaboration, V. Tano, The underlying event in jet and minimum bias events at the Tevatron, http://arXiv.org/abs/hep-ph/0111412.

[54] Boomerang Collaboration, A. H. Jaffe et. al., Cosmology from Maxima-1, Boomerang and COBE/DMR CMB observations, Phys. Rev. Lett. 86 (2001) 3475-3479, http://arXiv.org/abs/astro-ph/0007333. 
[55] C. Pryke et. al., Cosmological parameter extraction from the first season of observations with DASI, Astrophys. J. 568 (2002) 46-51, http://arXiv.org/abs/astro-ph/0104490.

[56] T. J. Pearson et. al., The anisotropy of the microwave background to $l=3500$ : Mosaic observations with the Cosmic Background Imager, http://arXiv.org/abs/astro-ph/0205388.

[57] J. A. Rubino-Martin et. al., First results from the Very Small Array. IV: Cosmological parameter estimation, http://arXiv.org/abs/astro-ph/0205367. MNRAS submitted.

[58] S. B. A. Lewis, Cosmological parameters from CMB and other data: a Monte-Carlo approach, http://arXiv.org/abs/astro-ph/0205436.

[59] W. J. Percival et. al., Parameter constraints for flat cosmologies from CMB and 2dFGRS power spectra, http://arXiv.org/abs/astro-ph/0206256. MNRAS submitted.

[60] G. Belanger, F. Boudjema, A. Pukhov, and A. Semenov, MicrOMEGAs: A program for calculating the relic density in the MSSM, http://arXiv.org/abs/hep-ph/0112278.

[61] T. Moroi and L. Randall, Wino cold dark matter from anomaly-mediated SUSY breaking, Nucl. Phys. B570 (2000) 455-472, http://arXiv.org/abs/hep-ph/9906527.

[62] U. Chattopadhyay and P. Nath, Probing supergravity grand unification in the brookhaven $g-2$ experiment, Phys. Rev. D53 (1996) 1648-1657, http://arXiv.org/abs/hep-ph/9507386.

[63] S. P. Martin and J. D. Wells, Muon anomalous magnetic dipole moment in supersymmetric theories, Phys. Rev. D64 (2001) 035003, http://arXiv.org/abs/hep-ph/0103067.

[64] S. Baek, P. Ko, and J. H. Park, Muon anomalous magnetic moment from effective supersymmetry, Eur. Phys. J. C24 (2002) 613-618, http://arXiv.org/abs/hep-ph/0203251.

[65] M. Byrne, C. Kolda, and J. E. Lennon, Updated implications of the muon anomalous magnetic moment for supersymmetry, http://arXiv.org/abs/hep-ph/0208067.

[66] M. Endo and T. Moroi, Muon magnetic dipole moment and higgs mass in supersymmetric SU(5) models, Phys. Lett. B525 (2002) 121-129, http://arXiv.org/abs/hep-ph/0110383.

[67] E821 Muon g-2 Collaboration, D. W. Hertzog, The BNL muon anomalous magnetic moment measurement, http://arXiv.org/abs/hep-ex/0202024. 
[68] E821 Muon g-2 Collaboration, G. W. Bennett, Measurement of the positive muon anomalous magnetic moment to 0.7 ppm, http://arXiv.org/abs/hep-ex/0208001.

[69] A. Czarnecki and W. J. Marciano, The muon anomalous magnetic moment: A harbinger for 'new physics', Phys. Rev. D64 (2001) 013014, http://arXiv.org/abs/hep-ph/0102122.

[70] F. J. Yndurain, Disagreement between standard model and experiment for muon g-2?, http://arXiv.org/abs/hep-ph/0102312.

[71] S. Narison, Muon and tau anomalies updated, Phys. Lett. B513 (2001) 53-70, http://arXiv.org/abs/hep-ph/0103199.

[72] J. F. de Troconiz and F. J. Yndurain, Precision determination of the muon $g_{\mu}-2$ and $\bar{\alpha}_{Q E D}\left(M_{Z}^{2}\right)$, http://arXiv.org/abs/hep-ph/0111258.

[73] M. Knecht and A. Nyffeler, Hadronic light-by-light corrections to the muon g-2: The pion-pole contribution, Phys. Rev. D65 (2002) 073034, http://arXiv.org/abs/hep-ph/0111058.

[74] M. Knecht, A. Nyffeler, M. Perrottet, and E. De Rafael, Hadronic light-by-light scattering contribution to the muon g-2: An effective field theory approach, Phys. Rev. Lett. 88 (2002) 071802, http://arXiv.org/abs/hep-ph/0111059.

[75] M. Hayakawa and T. Kinoshita, Comment on the sign of the pseudoscalar pole contribution to the muon g-2, http://arXiv.org/abs/hep-ph/0112102.

[76] J. Bijnens, E. Pallante, and J. Prades, Comment on the pion pole part of the light-by-light contribution to the muon g-2, Nucl. Phys. B626 (2002) 410-411, http://arXiv.org/abs/hep-ph/0112255.

[77] I. Blokland, A. Czarnecki, and K. Melnikov, Pion pole contribution to hadronic light-by-light scattering and muon anomalous magnetic moment, Phys. Rev. Lett. 88 (2002) 071803, http://arXiv.org/abs/hep-ph/0112117.

[78] J. L. Lopez, D. V. Nanopoulos, and X. Wang, Large $g_{\mu}-2$ in $S U(5) \times U(1)$ supergravity models, Phys. Rev. D49 (1994) 366-372, http://arXiv.org/abs/hep-ph/9308336.

[79] U. Chattopadhyay, D. K. Ghosh, and S. Roy, Constraining anomaly mediated supersymmetry breaking framework via ongoing muon $g-2$ experiment at brookhaven, Phys. Rev. D62 (2000) 115001, http://arXiv.org/abs/hep-ph/0006049.

[80] G. Degrassi, P. Gambino, and G. F. Giudice, $B \rightarrow X_{s} \gamma$ in supersymmetry: Large contributions beyond the leading order, JHEP 12 (2000) 009, http://arXiv.org/abs/hep-ph/0009337. 
[81] M. Ciuchini, G. Degrassi, P. Gambino, and G. F. Giudice, Next-to-leading QCD corrections to $B \rightarrow X_{s} \gamma$ : Standard Model and two-Higgs doublet model, Nucl. Phys. B527 (1998) 21-43, http://arXiv.org/abs/hep-ph/9710335.

[82] P. Gambino and M. Misiak, Quark mass effects in $\bar{B} \rightarrow X_{s} \gamma$, Nucl. Phys. B611 (2001) 338-366, http://arXiv.org/abs/hep-ph/0104034].

[83] CLEO Collaboration, S. Chen et. al., Branching fraction and photon energy spectrum for $b \rightarrow s \gamma$, Phys. Rev. Lett. 87 (2001) 251807, http://arXiv.org/abs/hep-ex/0108032.

[84] Belle Collaboration, Y. Ushiroda, Radiative B meson decay, http://arXiv.org/abs/hep-ex/0104045.

[85] BaBar Collaboration, B. Aubert et. al., Determination of the branching fraction for inclusive decays $B \rightarrow X_{s} \gamma$, http://arXiv.org/abs/hep-ex/0207076.

[86] P. Gambino and U. Haisch, Complete electroweak matching for radiative B decays, JHEP 10 (2001) 020, http://arXiv.org/abs/hep-ph/0109058.

[87] C. G. Lester and D. J. Summers, Measuring masses of semi-invisibly decaying particles pair produced at hadron colliders, Phys. Lett. B463 (1999) 99-103, http://arXiv.org/abs/hep-ph/9906349.

[88] UA1 Collaboration, G. Arnison et. al., Experimental observation of isolated large transverse energy electrons with associated missing energy at $\sqrt{S}=540$ GeV, Phys. Lett. B122 (1983) 103-116.

[89] I.Gavrilenko, Description of global pattern recognition program XKalman, ATL-INDET-97-165. ATLAS note.

[90] V. M. G. B. Epp and A. Nairz, A new hadronic-track parameterisation for fast simulation of the ATLAS inner detector, ATL-PHYS-2001-009. ATLAS note.

[91] T. Akesson et. al., Particle identification using the time-over-threshold method in the ATLAS transition radiation tracker, ATL-INDET-2000-021. ATLAS note. 